\begin{tabular}{|c|c|}
\hline Citation & $\begin{array}{l}\text { Jan De Munck, Atsushi Mine, André Poitevin, Annelies Van Ende, Marcio } \\
\text { Vivan Cardoso, Kirsten L Van Landuyt, Marleen Peumans, Bart Van } \\
\text { Meerbeek, (2012), } \\
\text { Meta-analytical Review of Parameters Involved in Dentin Bonding, } \\
\text { Journal of Dental Research, 91(4), 351-357. }\end{array}$ \\
\hline Archived version & $\begin{array}{l}\text { Author manuscript: the content is identical to the content of the published } \\
\text { paper, but without the final typesetting by the publisher }\end{array}$ \\
\hline Published version & http://dx.doi.org/10.1177/0022034511431251 \\
\hline Journal homepage & http://jidr.sagepub.com/ \\
\hline Author contact & $\begin{array}{l}\text { bart.vanmeerbeek@med.kuleuven.be } \\
+32(0) 16337587\end{array}$ \\
\hline IR & https://lirias.kuleuven.be/handle/123456789/346220 \\
\hline
\end{tabular}

(article begins on next page) 


\title{
Meta-analytical Review of Parameters Involved in Dentin Bonding
}

\author{
Jan De Munck, Atsushi Mine, André Poitevin, Annelies Van Ende, Marcio Vivan Cardoso, Kirsten L Van \\ Landuyt, Marleen Peumans, Bart Van Meerbeek
}

${ }^{1}$ KU Leuven BIOMAT, Department of Oral health Sciences, KU Leuven (University of Leuven), Belgium 


\begin{abstract}
Bond-strength testing is the most used method to asses bonding effectiveness to enamel and dentin. We aimed to disclose general trends in adhesive performance by collecting dentin bond-strength data systematically. The PubMed and Embase databases were used to identify 2157 bond-strength tests in 298 papers. Most used was the micro-tensile test, which appeared to have a larger discriminative power than the traditional macro-shear test. Because of the huge variability in dentin bond-strength data and the high number of co-variables, a neural network statistical model was constructed. Variables like 'research group' and 'adhesive brand' appeared most determining. Weighted means derived from this analysis confirmed the high sensitivity of current adhesive approaches (especially of all-in-one adhesives) to long-term waterstorage and substrate variability.
\end{abstract}

\title{
Key Words
}

meta-analysis, bond strength, macro-shear, micro-tensile, adhesive, dental materials 


\section{Introduction}

Worldwide, bond-strength tests are used to measure bonding effectiveness of adhesives to tooth tissue. Such tests serve multiple purposes, ranging from initial screening of new adhesive formulations, testing of various research hypotheses, up to quality control by manufacturers.

Many reviews have focused on bond strength and its predictability of clinical performance (Van Noort et al., 1989; Rueggeberg et al., 1991; Fowler et al., 1992; Pashley et al., 1995; Leloup et al., 2001). The main issues discussed in these reviews deal with the methodology employed and high data variability. The solution proposed was to standardize the bond-strength protocol, hoping for better data interpretation and in particular inter-study comparison (Van Noort et al., 1989; Stanley 1993). Today, the variety in test methodologies has however never been broader, while no consensus exists among researchers regarding the most appropriate use and interpretation of bond-strength data (Armstrong et al., 2010). Nevertheless, the amount of data currently available in literature is so vast that some relevant trends might pop up despite the high variance. This calls, however, for statistical techniques that can deal with such an exponentially growing pool of 'noisy' data (Bishop, 1995).

Therefore, the purpose of this study was to systematically collect dentin bond-strength data, to identify the primary parameters that affect the outcome of bond-strength tests, and to disclose trends in adhesive performance for the different adhesive approaches available today.

\section{Materials \& Methods}

\section{Systematic Literature Search}

By entering the search term 'dentin bond strength AND "published last 5 years"[Filter]' in PubMed, 1049 studies were identified. The search was conducted on January 17, 2009 and repeated on October 9, 2009. To identify manuscripts not listed in PubMed a similar search covering the same period was conducted using the EMBASE database. Subsequently, all papers, along with the respective identifiers, were inserted in a custom-made relational database. Original articles were retrieved and appraised by 4 calibrated researchers (JDM/AM/AP/AVE). Studies were included when the following criteria were met: (1) bond strength of at least 2 commercial adhesives to dentin was measured; (2) a light-curing composite (no resin cement, nor glass-ionomer) was employed; and (3) for every relevant experimental group in each study at least the following data were recorded: type of test, mean bond strength, standard deviation and number of specimens, teeth and pre-testing failures. Also, parameters regarding the test set-up were recorded, such as substrate origin and preparation, storage time and medium, adhesive and composite brand, light-curing methodology, potential aging procedure and some other test conditions like loading geometry and interface shape (Web appendix Tables 2 and 3).

From the PubMed/Embase identifiers, the number of studies present in the database for every author was calculated. In each study, the author with most studies in the database was identified and served as identifier for the 'research group' that generated the data. All adhesives were categorized according to the classification by Van Meerbeek et al. (2003). Additionally, data regarding composition and mechanical properties of adhesives $(\mathrm{pH}$, bonding technique,...) as well as composites (flexural modulus, composite type,... ) were retrieved from Van Landuyt et al. (2007) and Ilie and Hickel (2009), respectively. Further, to assess the effect of progress in adhesive dentistry, the first appearance in the PubMed database was recorded for each adhesive and is referred to as 'adhesive age'. 
To assess publication bias, dentin bond-strength data produced in a single dental materials research lab from the Katholieke Universiteit Leuven (Leuven BIOMAT Research Cluster) during the period 2002-2010 were analyzed, being further referred to as the 'BIOMAT database'.

\section{Descriptive statistics and correlation analyses}

The overall mean bond strength (in $\mathrm{MPa}$ ) and histogram of each bond-strength methodology and adhesive class were calculated. Subsequently, these data were subjected to a first statistical analysis using ANOVA. To assess the importance of several 'continuous' parameters on the outcome of bond-strength tests, correlation analyses were performed on more homogenous subsets. A specific subset of data was therefore prepared, in which only 'control' bond-strength data were included, more specifically representing data derived from specimens that were prepared according to the manufacturers' instructions and tested between 1 day and 1 week, without any artificial aging or mechanical loading imposed, nor involving any modifications to the manufacturer's instructed adhesive procedure. Other subsets included bond-strength data measured upon 'aging by water-storage' or 'thermo-cycling'.

\section{Artifical Neural Networks (ANN)}

Next, a subset was prepared containing data of only micro-tensile bond-strength ( $\mu$ TBS) results (61\% of the data: 1314 records). From all parameters registered, 10 clinically and statistically relevant parameters (Table 1) were extracted/calculated to be included in the statistical model. A randomly chosen subset, containing $80 \%$ of the dataset, served to create multilayer perceptron neural networks with up to 40 hidden units (Statistica 9.0, StatSoft, Tulsa, OK, USA). The remaining part of the data served to train and test the statistical models by comparing the 'predicted' to the 'actual' reported bond strength. To validate the networks, they were additionally trained on 2 random subsamples. From the 1500 networks that were trained, 8 were retained electronically and from the 8 remaining ones, the 4 providing the most consistent results were retained by hand. Training, testing and validation performance of all retained networks, as measured by correlation between the target and predicted value, was $83 \%, 75 \%$ and $73 \%$, respectively. An important feature of neural networks is generalization, i.e. the ability to predict the outcome of data unknown to the model. If the performance of the network is consistently good on both the test and validation samples, then it is reasonable to assume that the network generalizes well on unseen data. We used this feature to predict the performance of the 10 most common adhesives in the database, as tested by the most prevalent 'research groups'. In this way, a homogenous dataset was created, so that different groups could be compared.

\section{Results}

\section{Descriptive Statistics and Correlation Analyses}

From the 1049 studies identified, 298 yielded bond-strength data relevant for the current meta-analysis, from which 2157 individual bond-strength tests were extracted (Fig. 1). The two main reasons for exclusion were either that the manuscript contained no bond-strength data or that less than two commercial adhesives were used. Six major bond-strength tests were identified, namely 'tensile', 'shear' and 'push-out', each in a 'micro' and 'macro' variant. The $\mu$ TBS and macro-shear bond-strength (MSBS) tests together 
represented $83 \%$ of all tests (Fig. 1). Therefore, further analysis was limited to these 2 set-ups and was conducted separately, given the considerably different bond strength (Fig. 1) and loading conditions.

Two-way ANOVA indicated that 'adhesive class' as well as 'artificial aging' significantly affected the $\mu$ TBS outcome (Fig. 2). For the MSBS, only aging had a significant effect. Consequently, the discriminative power of a micro-tensile test appeared better than that of a macro-shear test. To substantiate this observation further, the 'control' bond strength of 2 frequently tested adhesives (Clearfil SE Bond, Kuraray and Adper Prompt L-Pop, 3M ESPE) was investigated in detail. $\mu$ TBS testing revealed a distinct and significant difference between both adhesives $(p<0.0001)$, while for the MSBS test, the relative difference was smaller and non-significant $(\mathrm{p}=0.3716)$.

Most correlations observed were low (highest $r=0.28$ ) and non-significant (Table 2). For the $\mu$ TBS subset, bond strength decreased significantly with increasing water-storage time (Table 2). To assess bond durability water-storage was used in 51 of the 295 retained studies. For the macro-shear subset, bond strength also decreased with storage time but not significantly. Thermo-cycling is the second most-used artificial aging methodology (39 studies), although 16 studies did not have a control group without thermocycling. The number of thermo-cycles, varying between 300 and 100,000, did however not affect bond strength (Table 2).

The BIOMAT database contains only $\mu$ TBS data, in majority to bur-cut dentin. The methodology varied only slightly over the years and only a limited amount of operators (<20) were involved. This resulted in a more consistent database than obtained from literature, but less representing the whole spectrum of experimental conditions. As the same inclusion criteria were employed as for the literature review, only 197 out of the 969 records contained relevant data, of which the majority was short-term bond-strength to burcut dentin. Some relevant data regarding extended water-storage and thermo-cycling were gathered as well (Fig. 3, web appendix Table 1). Overall a similar trend was observed as in the micro-tensile subset of the literature review (Table 3), though some differences were more pronounced. So is the bond strength of Adper Prompt 37\% lower than the three-step gold standard as predicted by the ANN, while in the BIOMAT database it is $48 \%$ lower.

\section{Artifical Neural Networks (ANN)}

The aim of the sensitivity analysis (Table 1) was to classify the meta-model input parameters according to their influence on the predicted dentin bond strength (the higher the value, the more relevant). The two variables most determining for the bond-strength outcome were the 'research group' and the 'adhesive' (2.9 and 2.81, respectively); both had almost three times more impact on bond strength than the least important variables: 'storage time', 'thermo-cycling' and 'age of the adhesive' (1.07, 1.02 and 1.02 respectively). Mean bond strength was predicted for the most prevalent adhesives tested in 'control' conditions (per manufacturer's instructions, bonded to SiC-paper ground dentin and tested within 1 week) and as it would be measured by the 7 most productive 'research groups' (Table 3). The mean bond strength of the best performing adhesive was almost twice as high as that of the least performing adhesive. Noteworthy is also that the range of predictions is very wide, as also appears from literature. For example, the 'control' bond strength reported in literature for OptiBond FI (Kerr) and One-up Bond F (Tokuyama) ranges from 33-81 $\mathrm{MPa}(\mathrm{n}=31)$ and from 11-51 MPa $(n=20)$, respectively. This range is in line with the ANN-predicted range of 39-58 MPa and 14-43 MPa, respectively. Similarly, the bond strength as would be obtained after one-year water storage or when the adhesive was bonded to dentin prepared by a diamond bur (as opposed to SiCpaper), was predicted using ANN. Both consistently resulted in a decrease, ranging from 5 to $39 \%$, in comparison to 'control' bond-strength data (Table 2). The magnitude of this decrease differed for each 
adhesive. Some adhesives were more affected by water storage, others more by bur preparation. Noteworthy is that adhesives with a higher 'control' bond strength, appeared less sensitive to aging. From the 10 most frequently tested adhesives, the highest predicted 'control' bond strength was recorded for OptiBond FL (Table 3); it appeared also least affected by aging and bur preparation (10\% and 5\% decrease, respectively). A little lower 'control' bond strength was recorded for the mild self-etch adhesive Clearfil SE Bond (Kuraray), for which the predicted decreases were 15\% after aging and 21\% when applied to bur-cut dentin. The lowest 'control' bond strength and highest bond-strength reduction (upon aging and bur-cut preparation) was recorded for One-up Bond F. A similar tendency was noted for other all-in-one adhesives.

When the adhesives that were most frequently tested in literature, were grouped per 'adhesive class' (Van Meerbeek et al., 2003), the highest and most aging-resistant bond strength was found for the 2-step selfetch adhesives, closely followed by the 3-step etch\&rinse adhesives (Table 3). Although the 'control' bond strength of the 2-step etch\&rinse adhesives were in the same range, they were much more affected by aging. One-step self-etch adhesives were affected considerably by aging as well as by bur-cut preparation.

\section{Discussion}

In this review, 295 bond-strength studies were included, considerably more than the 75 studies in a literature review conducted 10 years ago (Leloup et al., 2001, review period 1992 - 1996), even with our stricter inclusion criteria. In this meta-analysis, studies testing only a single commercial adhesive were excluded, while they made up 32\% of the data in the previous review. Given the enormous amount of data included, and for instance the fact that 7 out of the 10 most tested adhesives (Table 2) are at this time of publication still commercially available, and that very recent "state-of-the-art" assessments of literature (Pashley et al., 2011; Van Meerbeek et al., 2011) revealed no significant advancement in adhesive technology, nor in bond-strength study design, it is very likely that the outcome of this systematic analysis will remain relevant for quite some time to come. Two major test set-ups were present in literature, of which the micro-tensile test had a higher discriminative power than the macro-shear test. This may explain the current popularity of $\mu$ TBS testing in the research community: in the period 1992-1996 macro-shear testing still provided $75 \%$ of the data reported (Leloup et al., 2001), while this dropped to $22 \%$ in this study (20042009). This is substantiated by the observation that macro-shear testing apparently has no value in prediction of clinical performance (Heintze and Rousson, 2011), in contrast to the $\mu$ TBS that correlated with the 2-year and 5-year outcome of Class-V clinical studies (Van Meerbeek et al., 2010; Heintze and Rousson, 2011).

Statistical analysis of this meta-analytical review was based on ANN. This methodology is inspired on the working mechanism of the human brain and its principles are based on crude and low-level models of biological neural information processing systems. Neural networks have a remarkable ability to derive and extract meaning, rules, and trends from complicated, noisy, and imprecise data (Bishop, 1995). This neural network model-building technique is an alternative to more traditional statistical methods like general linear models and is getting popular not only in research, but also in fields as engineering and marketing (Paliwal and Kumar, 2009). Recently, ANN has been used to assess meta-analysis data in clinical psychology from 'dot-probe' tests conducted by diverse researchers (Frewen et al., 2008). A similar network-building technique was used to disclose interdependencies between brain regions from functional MRI imaging data collected from more than 500 imaging studies (Neumann et al., 2010). This demonstrates the versatility and wide range of applications for these ANN, especially for the analysis of a large amount of heterogeneous data. Generalization of the results of any of these models, i.e. to predict the outcome of tests not available 
in the database, is possible in case a proven methodology is followed (Kleijnen and Sargent, 2000) and when appropriate validation took place. Therefore, in this study, a part of the data (a random subset with $20 \%$ of the original data) was masked from the model fitting algorithm and only used to validate the observed data. This also allows controlling over- and under-fitting of the model to some degree.

The most significant parameter identified by ANN was the 'research group', which can be regarded as an aggregate parameter for all variables that are (hopefully more or less) standardized within one testing laboratory. Additionally, parameters included in the current analysis, like composite flexural modulus and substrate origin, are standardized in many research groups, which may reduce the sensitivity of these parameters, hence the reported effect is smaller than their real effect on the bond-strength outcome. This is also the main reason why experimental parameters, such as crosshead speed, clamping method, storage solution, specimen shape, etc. were not recorded; within each research group all these experimental parameters are standardized to enable intra-study comparison. For inter-study comparison, as primarily aimed with this review, test variability induced by specimen preparation and testing protocols cannot be neglected (Rueggeberg et al., 1991; Betamar et al., 2007, Poitevin et al., 2007). Different sources of variability can be identified: biological substrate variability (Shono et al., 1999) and substrate processinginduced differences, as for example different kinds of smear layer, specimen size and preparation (Oliveira et al., 2003; Van Meerbeek et al., 2003; Cardoso et al., 2008; Ermis et al., 2008). Even minute modifications of the test set-up can change the outcome considerably, as suggested by finite element analysis and practical testing (Rueggeberg et al., 1991; Fowler et al., 1992; Betamar et al., 2007; Poitevin et al., 2007). Given the heterogeneity of test results collected worldwide, inter-study comparison may appear useless (Scherrer et al., 2010). However, from this big amount of blurred data we were able to disclose some trends in bonding effectiveness to dentin using an ANN approach. The parameter 'research group' accounted for most of the variability in bonding effectiveness and therefore appeared most determining for the outcome. As a result, our 'predicted' bond-strength values largely varied, similar to bond strengths published in literature (Scherrer et al., 2010). But unlike literature, our 'predicted' bond-strength values are equally distributed and thus mutually comparable, thereby better representing real adhesive performance. An example in the BIOMAT database of this effect is that two-step self-etch adhesives apparently do not degrade by water storage (Web appendix Table 1). This has however partly to be attributed to the fact that in the water storage group, only very few groups, with better performing adhesives (Clearfil SE and Clearfil Protect Bond) are present. Therefore, these grouped performances are less reliable, when analyzed by conventional techniques, while by ANN this poses less of a problem.

A major concern in any meta-analysis is bias of the retrieved data at study level as well as at the outcome level (publication bias). Unlike clinical studies, randomization and blinding is uncommon for bond-strength studies and therefore no measure for the quality of the included studies. Therefore, also a guideline as the PRISMA statement (Moher et al., 2009), which is aimed at clinical interventions, is less applicable to the current review (Web appendix Table 4). Quality of the included studies was preserved by applying strict inclusion criteria. At study level, bias was further minimized by the fact that mostly 'control' data were used and the actual experimental groups (involving experimental compositions/conditions), more prone to reporting bias, were often not included in the database. To assess publication bias, data of the literature review were compared to data obtained in a single research lab. Overall, the similarities are prevailing. The two best performing adhesives are exactly the same; overall one-step self-etch adhesives do underperform and are prone to degradation upon water storage; adhesive performance is affected by water storage, while thermo-cycling has a negligible effect. Noteworthy is that in the BIOMAT database differences induced by experimental conditions are more pronounced (e.g. 1-year water storage induces a decrease of up to $72 \%$, while only up to $36 \%$ is predicted by ANN). This must be attributed to the attenuating effect of pooling many 
studies and the specific statistics employed that appear to result in a more conservative outcome. So overall, the effect of publication bias is minimal, apart from a publication delay as exemplified by the nonexistence of self-adhesive filling materials in literature, while these are already available in the BIOMAT database.

The best performing adhesive at both short and long term was the three-step etch\&rinse adhesive OptiBond FL (Kerr). This so-called 'gold standard' adhesive indeed presents with a very favorable laboratory (Peutzfeldt and Asmussen, 2006; Sarr et al., 2009) and clinical (Boghosian et al., 2007; Peumans et al., 2011) performance. The second best performing adhesive in this meta-analysis was the two-step self-etch adhesive Clearfil SE Bond (Kuraray), a mild self-etch adhesive, which is also reputed for its excellent long-term laboratory as well as clinical performance (Peumans et al., 2007; Osorio et al., 2008).

Major clinical concerns regarding today's adhesive technology are technique sensitivity and bond durability (Van Meerbeek et al., 2005), which both were assessed in this meta-analysis. In $16 \%$ of the database records, some aspects regarding technique sensitivity of adhesives were tested, such as increased/decreased etching time, adapted hydration state of the etched dentin, application of additional layers, increased/reduced air blowing, etc.. None of these adapted application protocols were, however, consistently applied by multiple authors so that a numerical appreciation was not possible. Pooling of all these modifications revealed only a very small reduction in bond strength, as compared to 'control' data, applied as per manufacturers' instructions (31.3 versus $31.5 \mathrm{MPa}$, respectively). One specific parameter is related to the way dentin is prepared prior to bonding. Most used preparation methods identified in this review were preparation by either a carbide or diamond dental bur or by silicon-carbide (SiC) paper. Overall, etch\&rinse adhesives appeared less affected when prepared by a dental bur (versus SiC-paper), while self-etch adhesives appeared more affected, especially the one-step version. This is not unexpected, because a bur-cut dentin surface is covered with a thicker and more compact smear layer (Oliveira et al., 2003) and thus may impede the bonding effectiveness of especially (ultra)-mild self-etch adhesives (Koshiro et al., 2006). It should be stressed that in simulation of what is clinically done, dentin is best prepared by bur, although in many bond-strength studies adhesives are bonded to SiC-ground dentin.

Regarding bond durability, more consensus in assessment methodology is present in literature. In $92 \%$ of the durability data, thermo-cycling or extended water storage was applied. Therefore, both methodologies were included in the ANN analysis as well as in the correlation analysis. Because of methodological issues, the sensitivity of both parameters was not very high in the ANN analysis, but some interesting conclusions can be drawn based upon correlations as well as predictions. First, 'thermo-cycling' did not affect the outcome of bond-strength tests, as was also shown in the previous review (Leloup et al., 2001). Partially, this may be due to the low number of thermo-cycles employed in most studies (mean/median was $6657 / 1150$ cycles). Simple 'water storage' has, however, a clear bond-degrading effect; it mimics the clinically observed restoration degradation very well (Hashimoto et al., 2000; De Munck et al., 2005). Our correlation analysis indicated that $\mu$ TBS degrades significantly $(p<0.0001)$ upon water storage. Neural network predictions do however point out that different adhesives degrade differently; bond-strength reductions ranged from 10 to 35\% (Table 3). The actual composition of the different adhesives, particularly the monomers, solvents and initiators used, may account for this observed variability. Data in the BIOMAT database appear more affected by water storage (Web appendix Table 1), which is, apart from methodological issues, also related to the storage of micro-specimens (direct exposure to water) instead of the whole restored teeth (indirect exposure) in most literature. Not surprisingly, especially three-step etch\&rinse and two-step self-etch adhesives appear most resistant to hydrolytic degradation; they provide a separate, more hydrophobic resin layer as final application step. 
In summary, despite the lack of a standard bond-strength protocol, this meta-analysis allowed, thanks to the vast amount of data available, to draw some clear conclusions with regard to the bonding effectiveness of different adhesive approaches to dentin. Moreover, there exists a definite need to measure not only the 'immediate' bond strength, but also 'aged' bond strength in prediction of long-term clinical performance. Finally, in simulation of a clinically produced smear layer, a dental bur should be preferentially used in laboratory bond-strength testing.

\section{Acknowledgments}

This study was supported by K.U.Leuven Research Grant OT/06/55. Dr. Van Landuyt is appointed as a Post-doctoral Research Fellow of FWO-Flanders. The authors declare that they have no conflict of interest regarding the products herein investigated. A preliminary report was presented at the IADR General session in Barcelona Spain (july 14-17, 2010). 


\section{REFERENCES}

Armstrong S, Geraldeli S, Maia R, Raposo LH, Soares CJ, Yamagawa J (2010). Adhesion to tooth structure: a critical review of "micro" bond strength test methods. Dent Mater 26:e50-62.

Betamar N, Cardew G, Van Noort R (2007). Influence of specimen designs on the microtensile bond strength to dentin. J Adhes Dent 9:159-168.

Bishop C. (1995). Neural Networks for Pattern Recognition. Oxford: University Press.

Boghosian AA, Drummond JL, Lautenschlager E (2007) Clinical evaluation of a dentin adhesive system: 13 year results. J Dent Res 86 (Spec Issue A): abstract 228.

Cardoso MV, Coutinho E, Ermis RB, Poitevin A, Van Landuyt K, De Munck J, Carvalho RCR, Van Meerbeek B (2008). Influence of dentin cavity surface finishing on micro-tensile bond strength of adhesives. Dent Mater 24:492-501.

De Munck J, Peumans M, Poitevin A, Lambrechts P, Braem M, Van Meerbeek B (2005). Durability of adhesion to tooth tissue: methods and results. J Dent Res 84:118-132.

Ermis RB, De Munck J, Cardoso MV, Coutinho E, Van Landuyt KL, Poitevin A, Lambrechts P, Van Meerbeek B (2008). Bond strength of self-etch adhesives to dentin prepared with three different diamond burs. Dent Mater 24:978-985.

Fowler CS, Swartz ML, Moore BK, Rhodes BF (1992). Influence of selected variables on adhesion testing. Dent Mater 8:265-269.

Frewen PA, Dozois DJ, Joanisse MF, Neufeld RW (2007).Selective attention to threat versus reward: metaanalysis and neural-network modeling of the dot-probe task. Clin Psychol Rev 28:307-337.

Hashimoto M, Ohno H, Kaga M, Endo K, Sano H, Oguchi H (2000). In vivo degradation of resin-dentin bonds in humans over 1 to 3 years. J Dent Res 79:1385-1391.

Heintze SD, Rousson V (2011). Pooling of dentin microtensile bond strength data improves clinical correlation. J Adhes Dent 13:107-110.

Ilie N, Hickel R (2009). Investigations on mechanical behaviour of dental composites. Clin Oral Investig 13:427-438

Kleijnen JPC, Sargent RG (2000). A methodology for fitting and validating metamodels in simulation. Eur J Oper Res 120:14-29.

Koshiro K, Sidhu SK, Inoue S, Ikeda T, Sano H (2006). New concept of resin-dentin interfacial adhesion: the nanointeraction zone. J Biomed Mater Res Part B: Appl Biomater 77B:401-408.

Leloup G, D'Hoore W, Bouter D, Degrange M, Vreven J (2001). Meta-analytical review of factors involved in dentin adherence. J Dent Res 80:1605-1614.

Moher D, Liberati A, Tetzlaff J, Altman DG, The PRISMA Group (2009). Preferred Reporting Items for Systematic Reviews and Meta-Analyses: The PRISMA Statement. PLoS Med 6:e1000097.

Neumann J, Fox PT, Turner R, Lohmann G (2010). Learning partially directed functional networks from meta-analysis imaging data. Neuroimage 49:1372-1384.

Oliveira SS, Pugach MK, Hilton JF, Watanabe LG, Marshall SJ, Marshall GW Jr (2003). The influence of the dentin smear layer on adhesion: a self-etching primer vs. a total-etch system. Dent Mater 19:758767.

Osorio R, Pisani-Proenca J, Erhardt MC, Osorio E, Aguilera FS, Tay FR, Toledano M (2008). Resistance of ten contemporary adhesives to resin-dentin bond degradation. J Dent 36:136-139.

Paliwala M, Kumar A (2009). Neural networks and statistical techniques: A review of applications. Expert Syst Appl 36:2-17.

Pashley DH, Sano H, Ciucchi B, Yoshiyama M, Carvalho RM (1995). Adhesion testing of dentin bonding agents: a review. Dent Mater 11:117-125.

Pashley DH, Tay FR, Breschi L, Tjäderhane L, Carvalho RM, Carrilho M, Tezvergil-Mutluay A (2011). State of the art etch-and-rinse adhesives. Dent Mater:1-16.

Peumans M, De Munck J, Van Landuyt K, Lambrechts P, Van Meerbeek B (2007). Five-year clinical effectiveness of a two-step self-etching adhesive. J Adhes Dent 9:7-10.

Peumans M, De Munck J, Van Landuyt K, Poitevin A, Lambrechts P, Van Meerbeek B (2011). A 13-year clinical evaluation of two three-step etch-and-rinse adhesives in non-carious class-V lesions. Clin Oral Invest (in press).

Peutzfeldt A, Asmussen E (2006). Influence of eugenol-containing temporary cement on bonding of selfetching adhesives to dentin. J Adhes Dent 8:31-34.

Poitevin A, De Munck J, Van Landuyt K, Coutinho E, Peumans M, Lambrechts P, Van Meerbeek B (2007). Influence of three specimen fixation modes on the micro-tensile bond strength of adhesives to dentin. Dent Mater J 26:694-699.

Rueggeberg FA (1991). Substrate for adhesion testing to tooth structure-review of the literature. Dent Mater 7:2-10.

Sarr M, Kane AW, Vreven J, Mine A, Van Landuyt KL, Peumans M, Lambrechts P, Van Meerbeek B, De Munck J (2009).Microtensile bond strength and interfacial characterization of 11 contemporary adhesives bonded to bur-cut dentin. Oper Dent 35:94-104.

Scherrer SS, Cesar PF, Swain MV (2010). Direct comparison of the bond strength results of the different test methods: a critical literature review. Dent Mater 26:e78-93. 
Shono Y, Ogawa T, Terashita M, Carvalho RM, Pashley EL, Pashley DH (1999). Regional measurement of resin-dentin bonding as an array. J Dent Res 78:699-705. Stanley HR (1993). An urgent plea for a standardized bonding (adhesion) test. J Dent Res 72:1362-1363.

Van Landuyt KL, Snauwaert J, De Munck J, Peumans M, Yoshida Y, Poitevin A, Coutinho E, Suzuki K, Lambrechts P, Van Meerbeek B (2007). Systematic review of the chemical composition of contemporary dental adhesives. Biomaterials 28:3757-3785.

Van Meerbeek B, De Munck J, Yoshida Y, Inoue S, Vargas M, Vijay P, Van Landuyt K, Lambrechts P, Vanherle G (2003). Buonocore memorial lecture. Adhesion to enamel and dentin: current status and future challenges. Oper Dent 28:215-235.

Van Meerbeek B, Van Landuyt K, De Munck J, Hashimoto M, Peumans M, Lambrechts P, Yoshida Y, Inoue S, Suzuki K (2005). Technique-sensitivity of contemporary adhesives. Dent Mater J 24:1-13.

Van Meerbeek B, Peumans M, Poitevin A, Mine A, Van Ende A, Neves A, De Munck J (2010). Relationship between bond-strength tests and clinical outcomes. Dent Mater 26:e100-21.

Van Meerbeek B, Yoshihara K, Yoshida Y, Mine A, De Munck J, Van Landuyt KL (2011). State of the art of self-etch adhesives. Dent Mater 27:17-28.

Van Noort R, Noroozi S, Howard IC, Cardew G (1989). A critique of bond strength measurements. J Dent $17: 61-67$. 


\section{FIGURES}

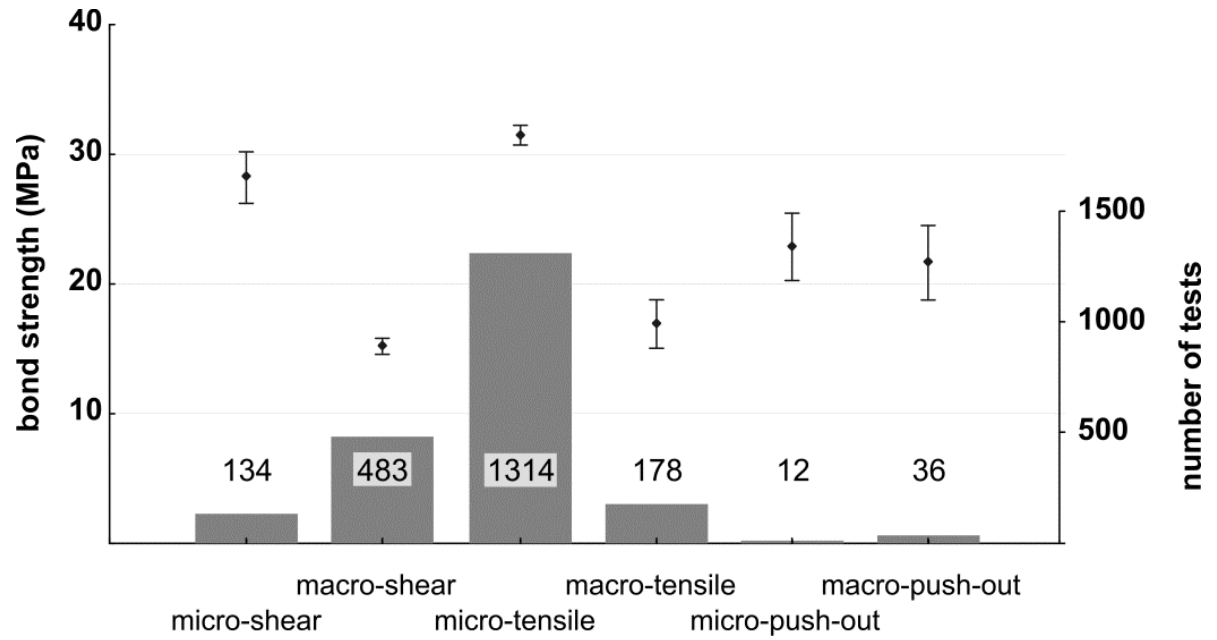

Fig. 1 Mean bond strength in MPa (whiskers denote 95\% confidence intervals), categorized for the different test set-ups (left axis), along with a histogram for the same set-ups (right axis).

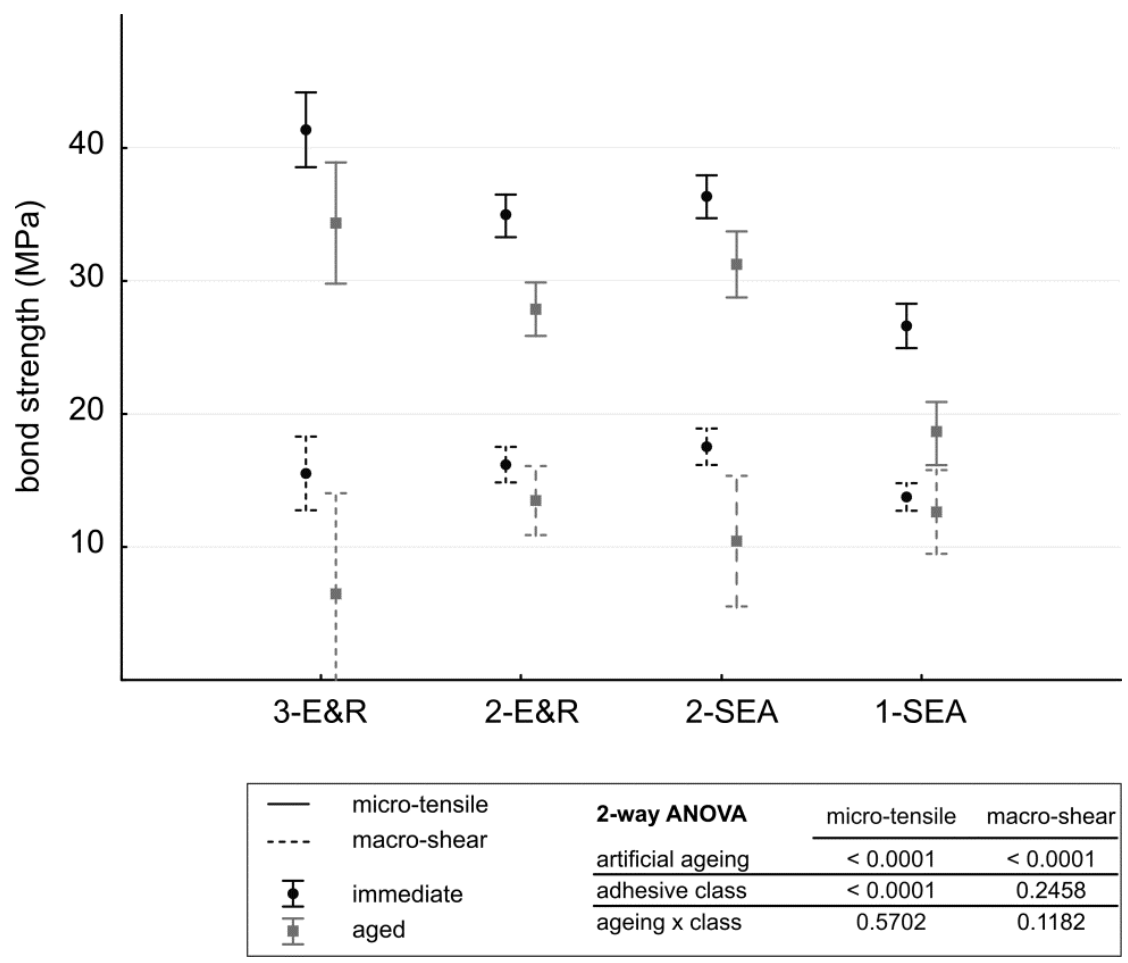

Fig. 2 Mean bond strength in MPa (whiskers denote 95\% confidence intervals) and ANOVA analysis for the two most common tests (micro-tensile and macro-shear), categorized per adhesive class (according to Van Meerbeek et al., 2003) and presence of artificial aging. Groups employing an adapted adhesive application were excluded from the analysis. 


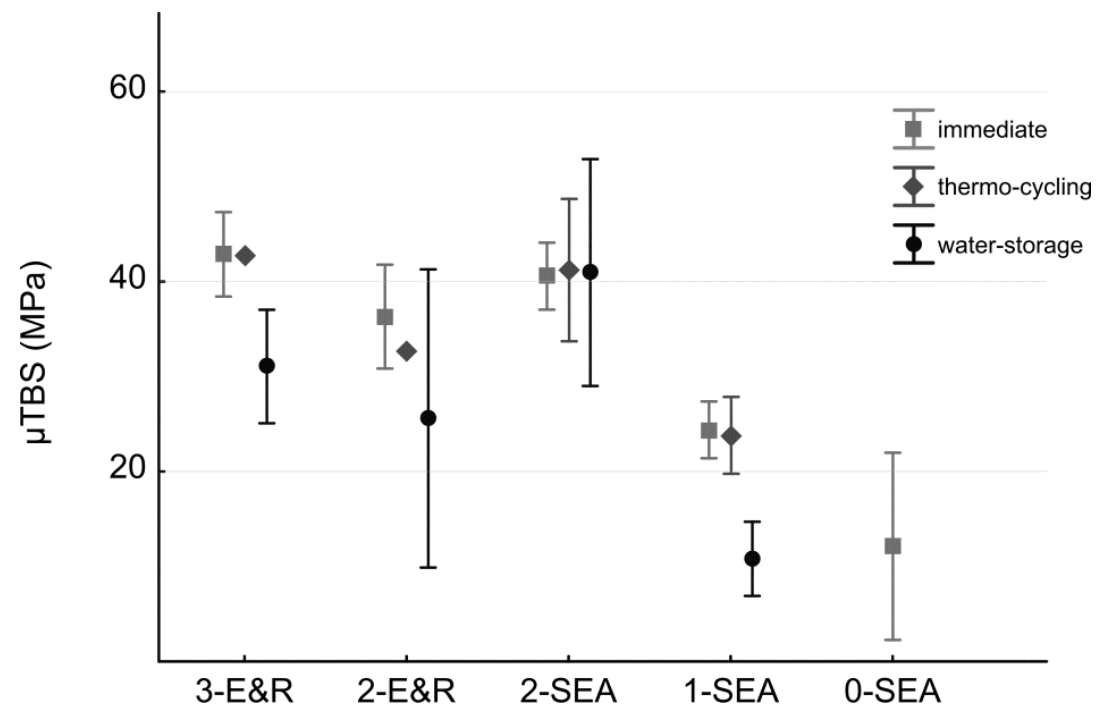

Fig. 3 Mean micro-tensile bond strength ( $\mu$ TBS) in MPa (whiskers denote 95\% confidence intervals) as obtained from the BIOMAT database, categorized by adhesive class (according to Van Meerbeek et al., 2003) and application of artificial aging. ${ }^{*} 0-S E A=0$-step self-etch adhesives or self-adhesive composite filling materials. 
TABLES

Table 1 Artificial network model parameters and average sensitivity of the retained models.

\begin{tabular}{lc}
\hline Model parameters & Mean sensitivity* \\
\hline research group & 2.90 \\
adhesive & 2.81 \\
control, aging or other & 2.16 \\
adhesive classification & 2.02 \\
substrate Preparation & 1.68 \\
substrate origin & 1.48 \\
composite flexural modulus & 1.11 \\
storage time & 1.07 \\
thermo-cycling & 1.02 \\
adhesive age & 1.02 \\
\hline
\end{tabular}

*The sensitivity values give an idea of the respective importance of these parameters in the statistical model.

Table 2 Correlation analyses.

\begin{tabular}{rcccc}
\hline & \multicolumn{2}{c}{ micro-tensile } & \multicolumn{2}{c}{ macro-shear } \\
& $\mathbf{r}$ & $\mathbf{p}$-value & $\mathbf{r}$ & $\mathbf{p}$-value \\
\hline storage time & -0.2793 & $<0.0001$ & -0.2791 & 0.0772 \\
thermo-cycling & 0.1413 & 0.1742 & 0.0078 & 0.9291 \\
composite flexural modulus & 0.1640 & 0.0001 & -0.0051 & 0.9444 \\
\hline age of adhesive & -0.1839 & $<0.0001$ & & \\
age of adhesive - 3-E\&R & 0.2474 & 0.0525 & & \\
age of adhesive - 2-E\&R & 0.0199 & 0.7775 & & \\
age of adhesive - 2-SEA & 0.0513 & 0.4832 & & \\
age of adhesive - 1-SEA & 0.2016 & 0.0049 & & \\
\hline
\end{tabular}

Pearson correlation coefficient and associated p-value of different parameters involved to measure the bond strength to dentin, as measured by a micro-tensile or macro-shear test set-up. correlation analyses for the different subsets of adhesive classes. 3-E\&R = 3-step etch\&rinse adhesives; 2-E\&R = 2-step etch\&rinse adhesives; 2-SEA = 2-step self-etch adhesives; 1-SEA = 1-step self-etch adhesives. 
Table 3 Bond strength (in MPa) as predicted by the artificial neural networks (ANN)

\begin{tabular}{|c|c|c|c|c|c|c|c|}
\hline & & \multicolumn{2}{|r|}{ SiC 1-day ${ }^{1}$} & \multicolumn{2}{|c|}{ Bur-cut 1-day² } & \multicolumn{2}{|c|}{ SiC 1-year 3} \\
\hline & & $n^{4}$ & $\mu$ TBS $^{5}$ & $\mu$ TBS $^{5}$ & decrease $^{6}$ & $\mu$ TBS $^{5}$ & decrease $^{6}$ \\
\hline ADHESIVE CLASS & 3-E\&R & 113 & $\begin{array}{c}41.7 \\
19.1-57.6\end{array}$ & $\begin{array}{c}36.1 \\
5.4-60.6\end{array}$ & $13 \%$ & $\begin{array}{c}34.6 \\
12-54.3\end{array}$ & $17 \%$ \\
\hline & 2-E\&R & 400 & $\begin{array}{c}39.1 \\
22.1-51.4\end{array}$ & $\begin{array}{c}34.4 \\
7.2-53.9\end{array}$ & $12 \%$ & $\begin{array}{c}27.8 \\
2.9-57.4\end{array}$ & $29 \%$ \\
\hline & 2-SEA & 281 & $\begin{array}{c}42.7 \\
23.6-56\end{array}$ & $\begin{array}{c}37.9 \\
6-60.1\end{array}$ & $11 \%$ & $\begin{array}{c}37.1 \\
21-51.9\end{array}$ & $13 \%$ \\
\hline & 1-SEA & 271 & $\begin{array}{c}34.4 \\
13.9-52.4\end{array}$ & $\begin{array}{c}25.2 \\
0.7-50.3\end{array}$ & $27 \%$ & $\begin{array}{c}23.2 \\
5.9-42\end{array}$ & $33 \%$ \\
\hline ADHESIVE & & & & & & & \\
\hline $\begin{array}{r}\text { Optibond FL } \\
\text { Kerr }\end{array}$ & $3-E \& R$ & 45 & $\begin{array}{c}49.7 \\
39.1-57.6\end{array}$ & $\begin{array}{c}47.3 \\
25.5-60.6\end{array}$ & $5 \%$ & $\begin{array}{c}44.8 \\
29.6-54.3\end{array}$ & $10 \%$ \\
\hline $\begin{array}{r}\text { Clearfil SE Bond } \\
\text { Kuraray }\end{array}$ & 2-SEA & 225 & $\begin{array}{c}45.4 \\
28.5-56\end{array}$ & $\begin{array}{c}35.8 \\
6.6-54.2\end{array}$ & $21 \%$ & $\begin{array}{c}38.6 \\
21-51.9\end{array}$ & $15 \%$ \\
\hline $\begin{array}{r}\text { Scotchbond } 1 \\
3 M \text { ESPE }\end{array}$ & $2-E \& R$ & 196 & $\begin{array}{c}42.2 \\
29.7-51.4\end{array}$ & $\begin{array}{c}34.4 \\
7.5-50.5\end{array}$ & $18 \%$ & $\begin{array}{c}30.9 \\
4.5-57.4\end{array}$ & $27 \%$ \\
\hline $\begin{array}{l}\text { Xeno III } \\
\text { Dentsply }\end{array}$ & 1-SEA & 46 & $\begin{array}{c}38.6 \\
29.1-48.3\end{array}$ & $\begin{array}{c}34.5 \\
11.9-50.3\end{array}$ & $11 \%$ & $\begin{array}{c}28.6 \\
16.4-42\end{array}$ & $26 \%$ \\
\hline $\begin{array}{l}\text { Adper Scotchbond Multi } \\
\text {-Purpose } 3 M \text { ESPE }\end{array}$ & $3-E \& R$ & 47 & $\begin{array}{c}38.4 \\
22.5-49.2\end{array}$ & $\begin{array}{c}31.5 \\
5.4-49.6\end{array}$ & $18 \%$ & $\begin{array}{c}30.3 \\
14.4-43\end{array}$ & $21 \%$ \\
\hline $\begin{array}{r}\text { Clearfil S }{ }^{3} \text { Bond } \\
\text { Kuraray }\end{array}$ & 1-SEA & 60 & $\begin{array}{c}37.8 \\
24.6-49.9\end{array}$ & $\begin{array}{c}26.9 \\
2.8-44.8\end{array}$ & $29 \%$ & $\begin{array}{c}26.1 \\
12.1-40.6\end{array}$ & $31 \%$ \\
\hline $\begin{array}{r}\text { One-Step } \\
\text { Bisco }\end{array}$ & 2-E\&R & 55 & $\begin{array}{c}36.3 \\
23.3-48.8\end{array}$ & $\begin{array}{c}32.8 \\
7.8-45.6\end{array}$ & $10 \%$ & $\begin{array}{c}23.2 \\
3.7-49.7\end{array}$ & $36 \%$ \\
\hline $\begin{array}{r}\text { Prime\&Bond NT } \\
\text { Dentsply }\end{array}$ & 2-E\&R & 45 & $\begin{array}{c}35.9 \\
22.1-46.3\end{array}$ & $\begin{array}{c}30.7 \\
7.2-46.2\end{array}$ & $15 \%$ & $\begin{array}{c}23.5 \\
2.9-44.2\end{array}$ & $35 \%$ \\
\hline $\begin{array}{r}\text { Adper Prompt L-Pop } \\
3 M \text { ESPE }\end{array}$ & 1-SEA & 61 & $\begin{array}{c}31.4 \\
18-43.9\end{array}$ & $\begin{array}{c}20.0 \\
1.3-39.6\end{array}$ & $36 \%$ & $\begin{array}{c}20.4 \\
8.1-34.4\end{array}$ & $35 \%$ \\
\hline $\begin{array}{r}\text { One-up Bond F } \\
\text { Tokuyama }\end{array}$ & 1-SEA & 44 & $\begin{array}{c}27.9 \\
13.9-42.8\end{array}$ & $\begin{array}{c}16.9 \\
0.7-34.8\end{array}$ & $39 \%$ & $\begin{array}{c}18.0 \\
5.9-34.9\end{array}$ & $35 \%$ \\
\hline
\end{tabular}

Mean predicted bond strength in MPa. Values reported for 'adhesive class' are the means pooled for all adhesives tested more than 15 times in the database. 'SiC 1-day' = immediate bond strength to SiC-ground dentin after 1-day water storage; 'Bur-cut 1-day' = immediate bond strength to bur-cut dentin after 1-day water storage; '3 SiC 1-year' = bond strength to SiC-ground dentin after 1-year water storage; ${ }^{4} \mathrm{n}=$ times tested in database; ${ }^{5} \mu \mathrm{TBS}=$ mean micro-tensile bond strength in $\mathrm{MPa}$, along with the the lowest and highest prediction for the respective adhesive class/adhesive in any of the 4 models as predicted by any of 7 'research groups'; ${ }^{6}$ Reduction in percentage versus the control (SiC 1-day) bond strength. 
WEB APPENDIX

Table 1 Micro-tensile bond-strength data of 'BIOMAT' database.

\begin{tabular}{|c|c|c|c|c|c|c|c|c|c|c|c|c|}
\hline \multirow[b]{2}{*}{ ADHESIVE CLASS $^{4}$} & & \multicolumn{3}{|c|}{ Bur-cut short-term ${ }^{1}$} & \multicolumn{4}{|c|}{ Bur-cut thermo-cycling ${ }^{2}$} & \multicolumn{4}{|c|}{ Bur-cut water storage $^{3}$} \\
\hline & & $\mu \mathrm{TBS}^{5}$ & $n^{6}$ & Std. err. $^{7}$ & $\mu$ TBS & $\mathbf{n}$ & Std. err. & $\%$ decrease $^{8}$ & $\mu$ TBS & n & Std. err. & $\%$ decrease \\
\hline 3-E\&R & & 42.9 & 25 & 2.2 & 42.8 & 2 & 5.7 & $0 \%$ & 31.1 & 7 & 2.4 & $28 \%$ \\
\hline 2-E\&R & & 36.3 & 16 & 2.6 & 32.6 & 1 & & $10 \%$ & 25.6 & 3 & 3.6 & $30 \%$ \\
\hline 2-SEA & & 40.6 & 48 & 1.8 & 41.2 & 7 & 3.1 & $-2 \%$ & 40.9 & 9 & 5.2 & $-1 \%$ \\
\hline 1-SEA & & 24.4 & 47 & 1.5 & 23.8 & 13 & 1.9 & $2 \%$ & 10.8 & 10 & 1.7 & $56 \%$ \\
\hline 0 -SEA & & 12.1 & 5 & 3.5 & & & & & & & & \\
\hline \multicolumn{13}{|l|}{ ADHESIVE } \\
\hline Optibond FL (Kerr) & 3-E\&R & 43.5 & 23 & 2.2 & 42.8 & 2 & 5.7 & $2 \%$ & 31.1 & 7 & 2.4 & $29 \%$ \\
\hline Clearfil SE Bond (Kuraray) & 2-SEA & 43.5 & 30 & 2.0 & 39.0 & 5 & 3.9 & $10 \%$ & 38.8 & 6 & 7.5 & $11 \%$ \\
\hline Clearfil Protect Bond (Kuraray) & 2-SEA & 42.6 & 5 & 3.4 & & & & & 45.3 & 3 & 5.4 & $-6 \%$ \\
\hline $\begin{array}{l}\text { Adper Scotchbond } 1 \text { XT (3M } \\
\text { ESPE) }\end{array}$ & 2-E\&R & 38.6 & 4 & 5.0 & & & & & 25.6 & 3 & 3.6 & $34 \%$ \\
\hline G-Bond (GC) & 1-SEA & 31.6 & 13 & 2.5 & 27.1 & 4 & 5.8 & $14 \%$ & 8.7 & 4 & 1.8 & $72 \%$ \\
\hline Bond Force (Tokuyama) & 1-SEA & 24.2 & 5 & 3.8 & & & & & & & & \\
\hline Adper Prompt L-Pop (3M ESPE) & 1-SEA & 22.5 & 4 & 4.8 & 21.2 & 2 & 3.0 & $6 \%$ & & & & \\
\hline iBond (Heraeus) & 1-SEA & 19.9 & 6 & 2.8 & 18.6 & 2 & 0.2 & $7 \%$ & 10.9 & 2 & 0.8 & $45 \%$ \\
\hline AdheSE One (Ivoclar-Vivadent) & 1-SEA & 10.0 & 5 & 0.7 & & & & & 9.0 & 3 & 1.5 & $10 \%$ \\
\hline
\end{tabular}

195 out of 972 tests in the database matched the inclusion criteria. ${ }^{1}$ 'Bur-cut short-term’ = immediate bond strength to diamond bur-cut dentin after 24 -hour or 1 -week of water storage; '“Bur-cut thermo-cycling' = bond strength to diamond bur-cut dentin after thermo-cycling for 1800 to 20000 cycles (10991 cycles on average); ${ }^{3}$ 'Bur-cut water storage' = bond strength diamond bur-cut dentin after 6-month or 1-year water storage. ${ }^{4}$ Values reported for 'adhesive class' are the means pooled for all adhesives in the database, adhesieves were categorized according to the classification of Van Meerbeek et al. (2003): 0-SEA = self-adhesive composites; 1-SEA = 1-step self-etch adhesives; 2-SEA = 2step self-etch adhesives; 2-E\&R = 2-step etch\&rinse adhesives; 3-E\&R = 3-step etch\&rinse adhesives; ${ }^{5}$ Weighted means in MPa; ${ }^{6}$ Times tested in the 'BIOMAT' database;

${ }^{7}$ Standard error of the mean; ${ }^{8}$ Reduction in percentage versus the control (immediate) bond strength.. $\mathrm{n}=$ times tested in database. 
Table 2 Parameters stored in the database for every manuscript.

\begin{tabular}{|c|c|}
\hline Field & example \\
\hline AB - PubmedID & This study evaluated the effect of $2 \%, \ldots$ \\
\hline AD - PubmedID & Department of Restorative Dentistry, ... \\
\hline$A U$ - PubmedID & Komori PC, ... \\
\hline DA - PubmedID & 20090414 \\
\hline DCOM - PubmedID & 20090812 \\
\hline DP - PubmedID & 2009 Mar-Apr \\
\hline IP - PubmedID & 2 \\
\hline IS - PubmedID & 0361-7734 (Print) \\
\hline JID - PubmedID & 7605679 \\
\hline Journal - PubmedID & Oper Dent \\
\hline JT - PubmedID & Operative dentistry \\
\hline $\mathrm{MH}$ - PubmedID & Acid Etching, Dental, ... \\
\hline OWN - PubmedID & NLM \\
\hline PG - PubmedID & $157-65$ \\
\hline PMID - PubmedID & 19363971 \\
\hline PT - PubmedID & Research Support, Non-U.S. Gov't \\
\hline RN - PubmedID & 0 (Composite Resins), ... \\
\hline SB - PubmedID & $\mathrm{D}$ \\
\hline SO - PubmedID & Oper Dent. 2009 Mar-Apr;34(2):157-65. \\
\hline STAT - PubmedID & MEDLINE \\
\hline TA - PubmedID & Oper Dent \\
\hline TI - PubmedID & Effect of $2 \%$ chlorhexidine, $\ldots$ \\
\hline VI - PubmedID & 34 \\
\hline ID of the study in the database & Study_ID_1345 \\
\hline Brand name of composite used & Filtek Z250 \\
\hline Type of light-curing unit & halogen \\
\hline Study involves extended water storage (yes/no) & yes \\
\hline Study involves thermocycling (yes/no) & no \\
\hline Study involves some other kind of aging (yes/no) & no \\
\hline Different storage periods (in months) & week, 6 \\
\hline Origin of the dentin substrate (human / bovine) & human \\
\hline Type of substrate & caries-affected dentin, dentin \\
\hline Preparation of the substrate prior to adhesive procedures & SiC paper \\
\hline Study involves alternative application technique (yes/no) & no \\
\hline Application techniques used & Instructions of manufacturer \\
\hline Number of teeth used per experimental group & 5 \\
\hline Size of test area in $\mathrm{mm}^{2}$ & 0.81 \\
\hline Test area (micro/macro) & micro \\
\hline Shape of the tested interface (square/round) & square \\
\hline Test mode (tensile/shear/push-out) & tensile \\
\hline $\begin{array}{l}\text { Author with highest number of citations in the database } \\
\text { Review_status }\end{array}$ & $\begin{array}{l}\text { Pashley } \mathrm{DH} \\
\text { reviewed }\end{array}$ \\
\hline
\end{tabular}


Table 3 Parameters stored in the database for every experimental group.

\begin{tabular}{ll}
\hline field & example \\
\hline ID of the experimental group in the database & Gr_2111 \\
ID of the study in the database & Study_ID_1345 \\
Size of test area in mm² & 0.81 \\
Size of test area (micro/macro) & micro \\
Test mode & tensile \\
Type of test & micro-tensile \\
Control group (yes/no) & no \\
Durability group (yes/no) & yes \\
Storage time in months & 6 \\
Number of thermocycles & 0 \\
Adhesive brand name & Scotchbond Multi-purpose \\
Adhesive brand name as looked up in database & Adper Scotchbond Multi-Purpose \\
Composite brand name & Filtek Z250 \\
Type of light-curing unit & halogen \\
Adhesive application technique emloyed & Instructions of manufacturer \\
Altered application technique employed (yes/no) & no \\
Mean bond strength in MPa & 28.0 \\
Number of specimens in the group & 48 \\
Number of pre-testing failures in this group & 2 \\
Standard deviation & 6 \\
Number of teeth used in this group & 5 \\
\hline
\end{tabular}




\subsection{Table 4 Prisma 2009 checklist}

\begin{tabular}{|c|c|c|c|}
\hline Section/topic & $\#$ & Checklist item & $\begin{array}{l}\text { Reported } \\
\text { on page \#* }\end{array}$ \\
\hline \multicolumn{4}{|l|}{ TITLE } \\
\hline Title & 1 & Identify the report as a systematic review, meta-analysis, or both. & 1 \\
\hline \multicolumn{4}{|l|}{ ABSTRACT } \\
\hline Structured summary & 2 & $\begin{array}{l}\text { Provide a structured summary including, as applicable: background; objectives; data sources; study eligibility } \\
\text { criteria, participants, and interventions; study appraisal and synthesis methods; results; limitations; conclusions and } \\
\text { implications of key findings; systematic review registration number. }\end{array}$ & A \\
\hline \multicolumn{4}{|l|}{ INTRODUCTION } \\
\hline Rationale & 3 & Describe the rationale for the review in the context of what is already known. & I, §2 \\
\hline Objectives & 4 & $\begin{array}{l}\text { Provide an explicit statement of questions being addressed with reference to participants, interventions, } \\
\text { comparisons, outcomes, and study design (PICOS). }\end{array}$ & $\begin{array}{l}\mathrm{M} \& \mathrm{M} \\
\S 1^{\star \star}\end{array}$ \\
\hline \multicolumn{4}{|l|}{ METHODS } \\
\hline Protocol and registration & 5 & $\begin{array}{l}\text { Indicate if a review protocol exists, if and where it can be accessed (e.g., Web address), and, if available, provide } \\
\text { registration information including registration number. }\end{array}$ & NA \\
\hline Eligibility criteria & 6 & $\begin{array}{l}\text { Specify study characteristics (e.g., PICOS, length of follow-up) and report characteristics (e.g., years considered, } \\
\text { language, publication status) used as criteria for eligibility, giving rationale. }\end{array}$ & M\&M, §1 \\
\hline Information sources & 7 & $\begin{array}{l}\text { Describe all information sources (e.g., databases with dates of coverage, contact with study authors to identify } \\
\text { additional studies) in the search and date last searched. }\end{array}$ & M\&M, §1 \\
\hline Search & 8 & $\begin{array}{l}\text { Present full electronic search strategy for at least one database, including any limits used, such that it could be } \\
\text { repeated. }\end{array}$ & M\&M, §1 \\
\hline Study selection & 9 & $\begin{array}{l}\text { State the process for selecting studies (i.e., screening, eligibility, included in systematic review, and, if applicable, } \\
\text { included in the meta-analysis). }\end{array}$ & M\&M, §1 \\
\hline
\end{tabular}




\begin{tabular}{|c|c|c|c|}
\hline Data collection process & 10 & $\begin{array}{l}\text { Describe method of data extraction from reports (e.g., piloted forms, independently, in duplicate) and any processes } \\
\text { for obtaining and confirming data from investigators. }\end{array}$ & M\&M, §1 \\
\hline Data items & 11 & $\begin{array}{l}\text { List and define all variables for which data were sought (e.g., PICOS, funding sources) and any assumptions and } \\
\text { simplifications made. }\end{array}$ & $\begin{array}{l}\text { WA, p. 3- } \\
4\end{array}$ \\
\hline $\begin{array}{l}\text { Risk of bias in individual } \\
\text { studies }\end{array}$ & 12 & $\begin{array}{l}\text { Describe methods used for assessing risk of bias of individual studies (including specification of whether this was } \\
\text { done at the study or outcome level), and how this information is to be used in any data synthesis. }\end{array}$ & D, §3 \\
\hline Summary measures & 13 & State the principal summary measures (e.g., risk ratio, difference in means). & $M \& M, \S 4$ \\
\hline Synthesis of results & 14 & $\begin{array}{l}\text { Describe the methods of handling data and combining results of studies, if done, including measures of consistency } \\
\left(\text { e.g., } I^{2}\right) \text { for each meta-analysis. }\end{array}$ & $M \& M, \S 5$ \\
\hline
\end{tabular}

\begin{tabular}{|c|c|c|c|}
\hline Section/topic & \# & Checklist item & $\begin{array}{l}\text { Reported } \\
\text { on page \# }\end{array}$ \\
\hline Risk of bias across studies & 15 & $\begin{array}{l}\text { Specify any assessment of risk of bias that may affect the cumulative evidence (e.g., publication bias, selective } \\
\text { reporting within studies). }\end{array}$ & D, §3 \\
\hline Additional analyses & 16 & $\begin{array}{l}\text { Describe methods of additional analyses (e.g., sensitivity or subgroup analyses, meta-regression), if done, indicating } \\
\text { which were pre-specified. }\end{array}$ & M\&M, §4 \\
\hline \multicolumn{4}{|l|}{ RESULTS } \\
\hline Study selection & 17 & $\begin{array}{l}\text { Give numbers of studies screened, assessed for eligibility, and included in the review, with reasons for exclusions at } \\
\text { each stage, ideally with a flow diagram. }\end{array}$ & $\mathrm{R}, \S 1$ \\
\hline Study characteristics & 18 & $\begin{array}{l}\text { For each study, present characteristics for which data were extracted (e.g., study size, PICOS, follow-up period) and } \\
\text { provide the citations. }\end{array}$ & NA \\
\hline Risk of bias within studies & 19 & Present data on risk of bias of each study and, if available, any outcome level assessment (see item 12). & D, §3 \\
\hline Results of individual studies & 20 & $\begin{array}{l}\text { For all outcomes considered (benefits or harms), present, for each study: (a) simple summary data for each } \\
\text { intervention group (b) effect estimates and confidence intervals, ideally with a forest plot. }\end{array}$ & NA \\
\hline Synthesis of results & 21 & Present results of each meta-analysis done, including confidence intervals and measures of consistency. & T3 \\
\hline Risk of bias across studies & 22 & Present results of any assessment of risk of bias across studies (see Item 15). & $\mathrm{D}, \S 3$ \\
\hline Additional analysis & 23 & Give results of additional analyses, if done (e.g., sensitivity or subgroup analyses, meta-regression [see Item 16]). & $\begin{array}{l}\text { WA P 5- } \\
8, \text { T2 }\end{array}$ \\
\hline
\end{tabular}




\section{DISCUSSION}

Summary of evidence

24 Summarize the main findings including the strength of evidence for each main outcome; consider their relevance to key groups (e.g., healthcare providers, users, and policy makers).

\begin{tabular}{|c|c|c|c|}
\hline & & & \\
\hline Limitations & 25 & $\begin{array}{l}\text { Discuss limitations at study and outcome level (e.g., risk of bias), and at review-level (e.g., incomplete retrieval of } \\
\text { identified research, reporting bias). }\end{array}$ & $D, \S 2,3$ \\
\hline Conclusions & 26 & Provide a general interpretation of the results in the context of other evidence, and implications for future research. & D, $\S 7$ \\
\hline FUNDING & & & \\
\hline Funding & 27 & $\begin{array}{l}\text { Describe sources of funding for the systematic review and other support (e.g., supply of data); role of funders for the } \\
\text { systematic review. }\end{array}$ & Ac \\
\hline
\end{tabular}

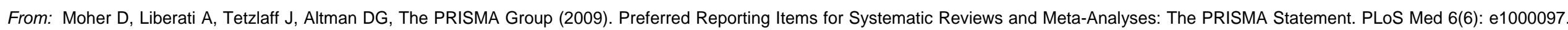
doi:10.1371/journal.pmed1000097

For more information, visit: www.prisma-statement.org.

${ }^{*} \mathrm{~A}=$ Abstract; I = Introduction; M\&M = Materials and Methods; R = Results; $\mathrm{D}=$ Discussion; Ac = Acknowledgements; WA = Web Appendix; $\mathrm{T}=$ Table and NA = Not

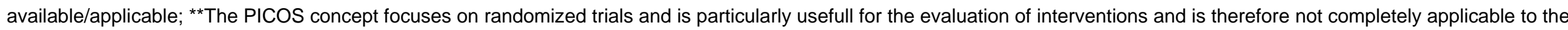
current review. 


\section{macro-shear}

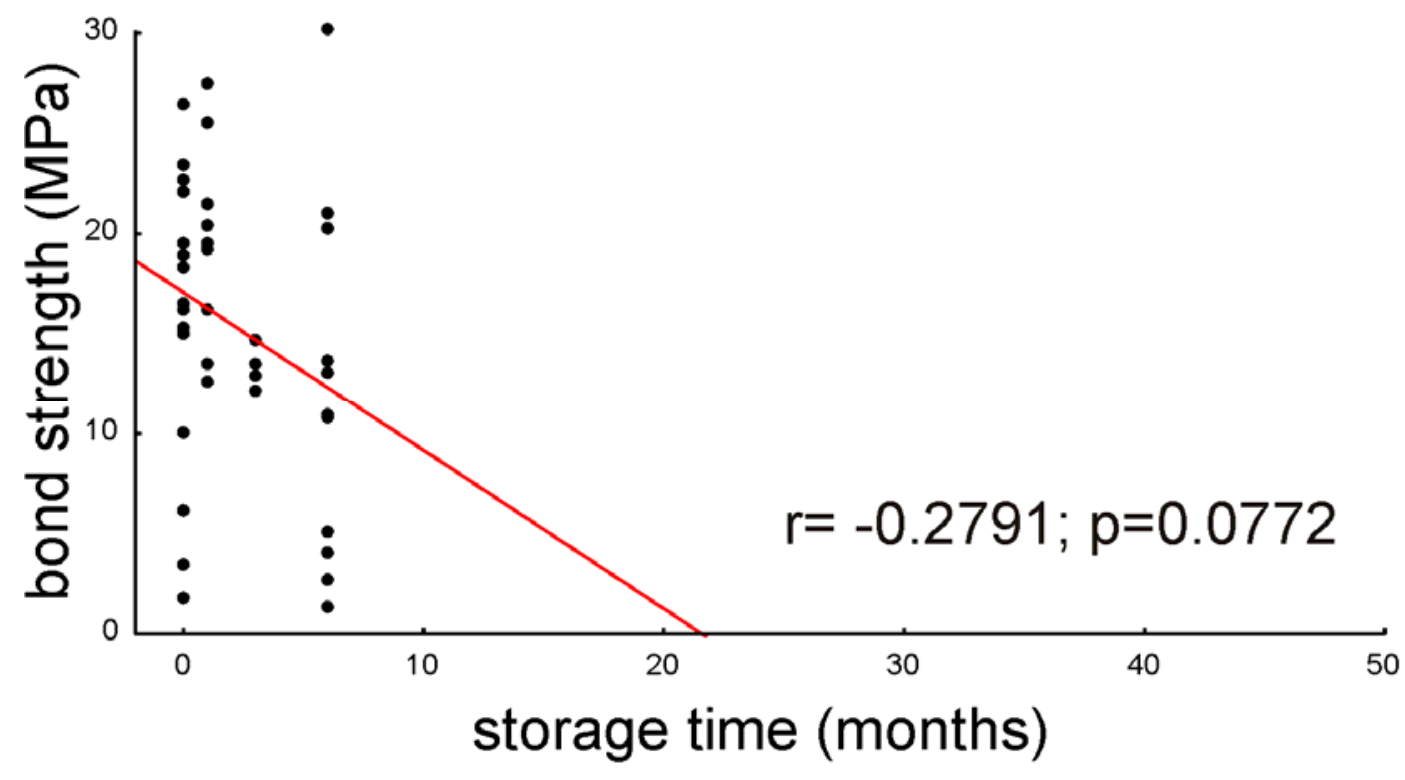

micro-tensile

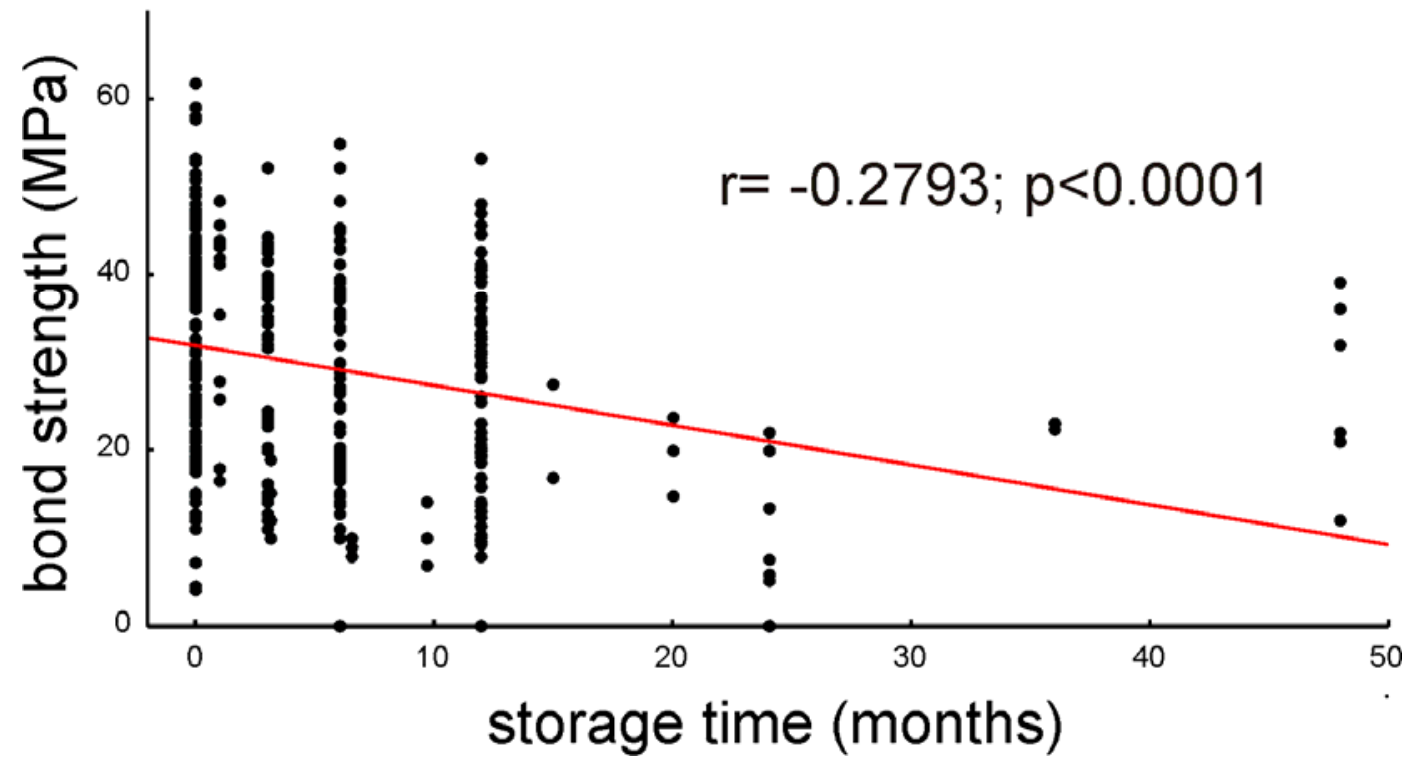

Figure 1 Correlation analysis - effect of water storage. The bond strength measured in studies employing extended water storage was plotted against the storage time used and categorized for the 2 most common tests (micro-tensile and macro-shear). 

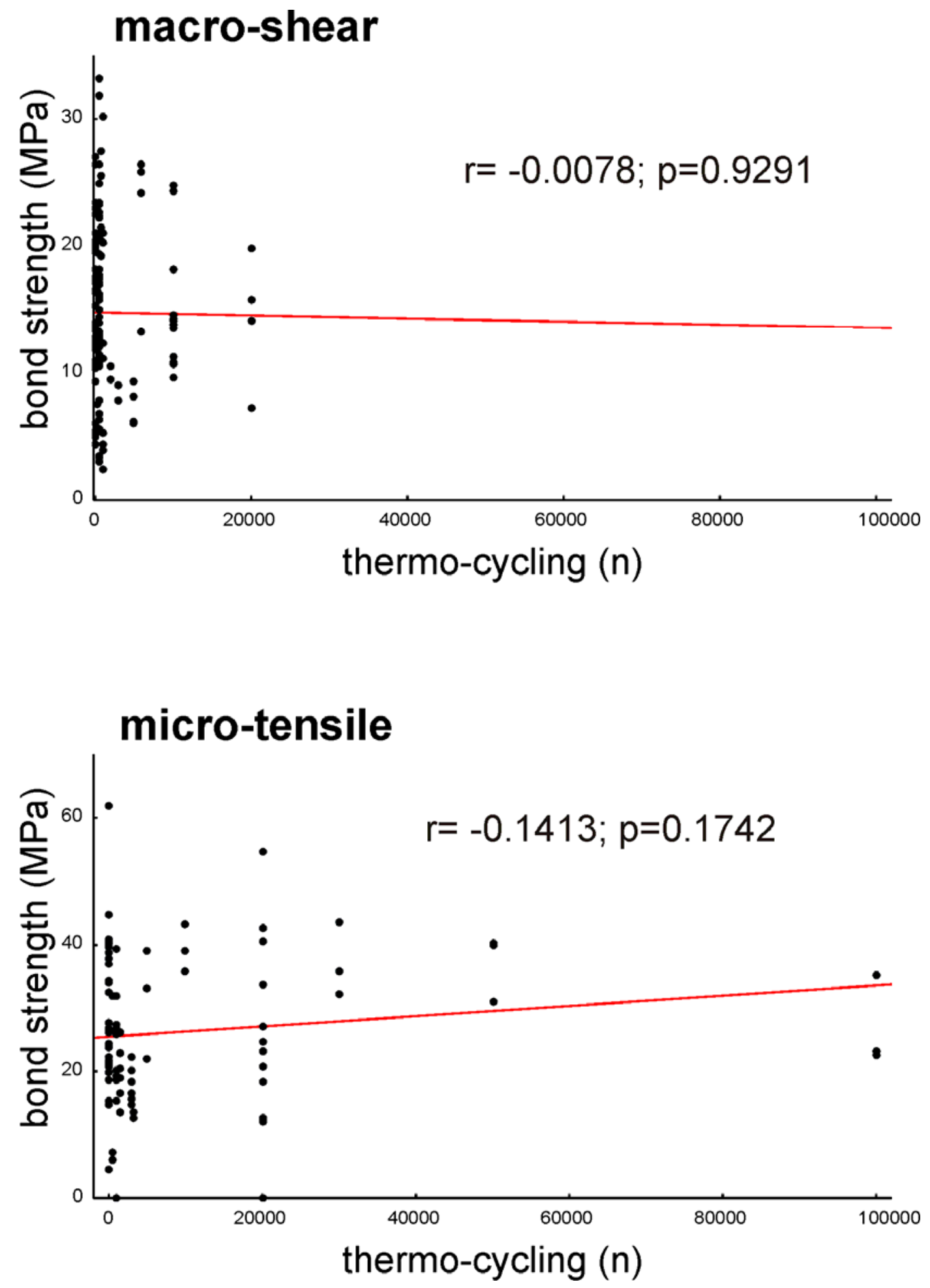

Figure 2 Correlation analysis - effect of thermo-cycling. The bond strength measured in studies employing thermo-cycling was plotted against the number of thermo-cycles used and categorized for the 2 most common tests (micro-tensile and macro-shear). As data for micro-tensile studies were not equally grouped (very few data with very long thermo-cycling), also the non-parametric Spearman rank correlation was calculated, but this correlation was also very small, negative and non-significant (-0.0735). 


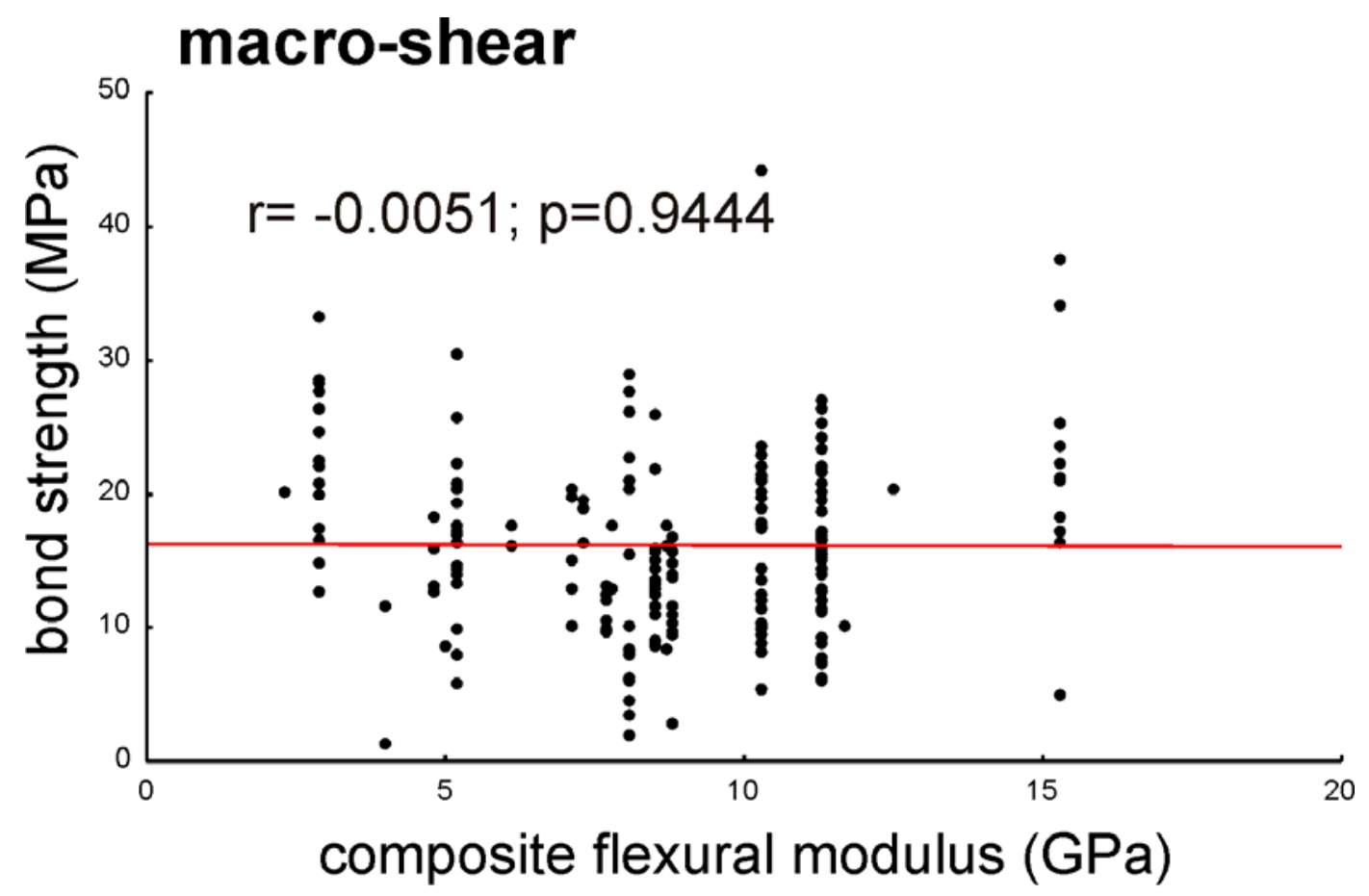

micro-tensile

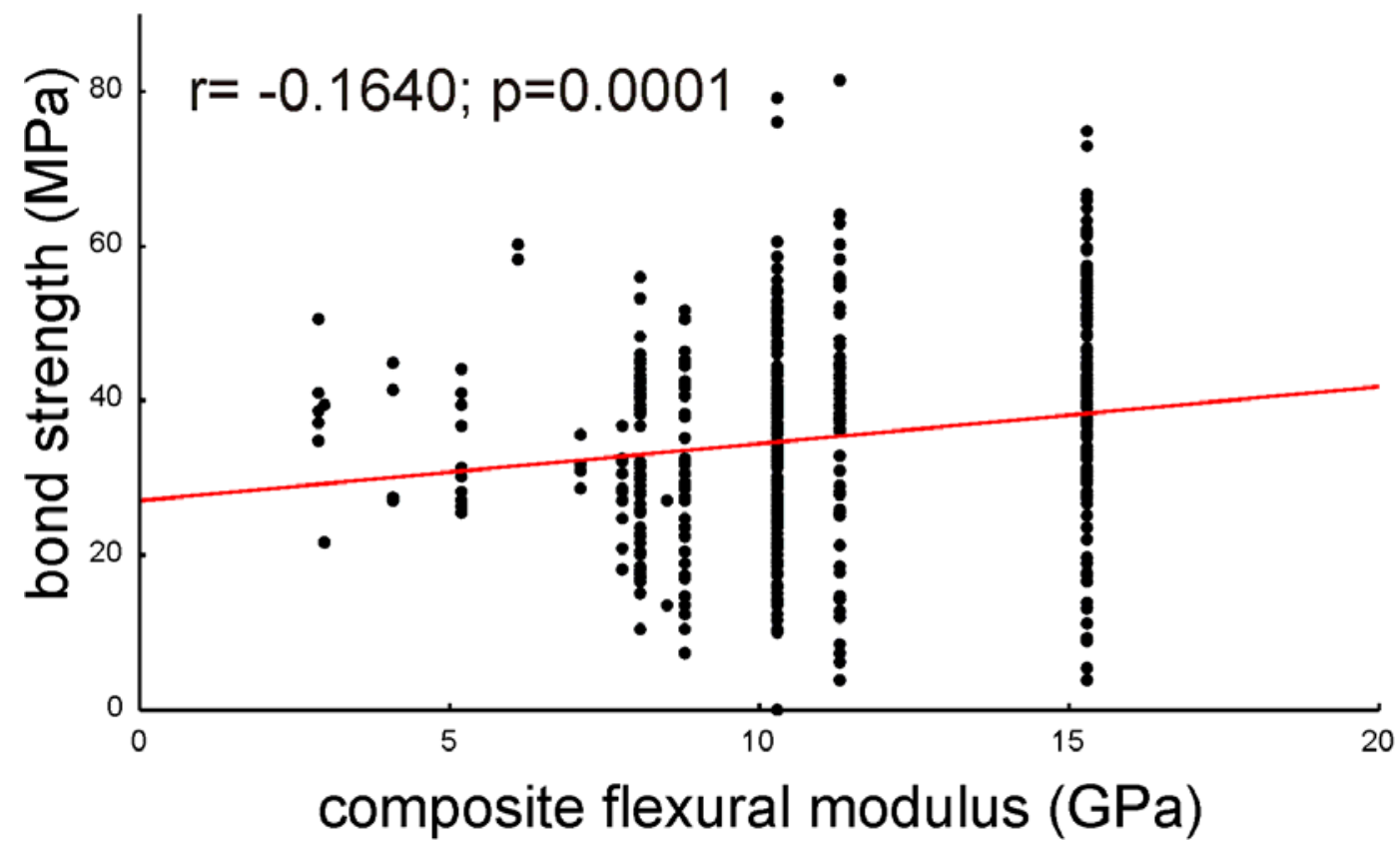

Figure 3 Correlation analysis - effect of composite. The bond strength of control (no aging, no other factors) tests was plotted against the composite flexural modulus (retrieved from llie et al. 2009) of the resin composite used in GPa and categorized for the 2 most common tests (micro-tensile and macro-shear). 

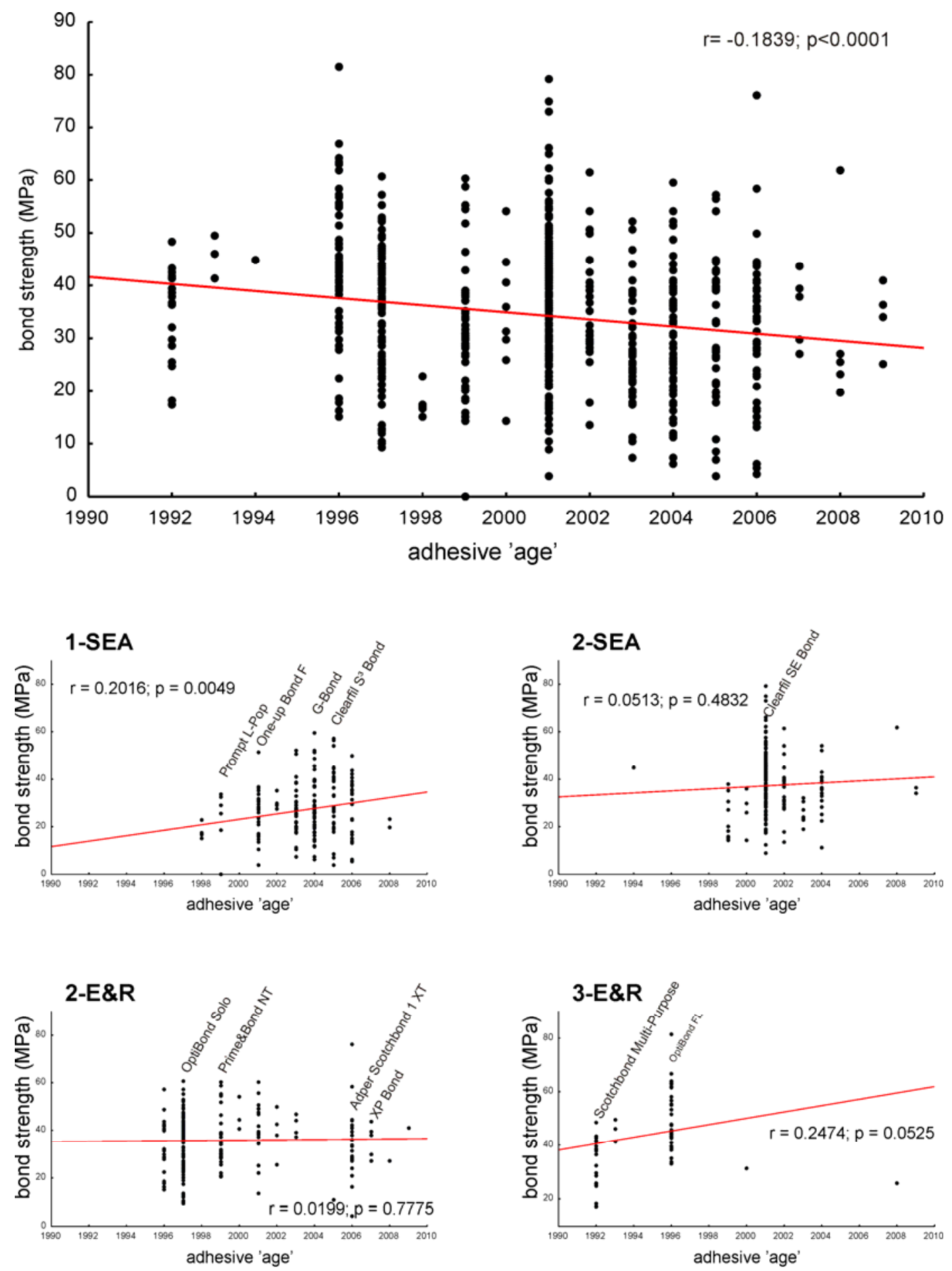

Figure 4 Correlation analysis - effect of adhesive 'age'. The micro-tensile bond strength of control (no aging, no other factors) tests was plotted against the year of first appearance in PubMed of the respective adhesive. Additionally the data were categorized by the adhesive approach (micro-tensile and macroshear). 
List of papers included in the meta-analysis.

1. Abdalla Al (2004). Microtensile and tensile bond strength of single-bottle adhesives: a new test method. J Oral Rehabil 31:379-384.

2. Abdalla Al, El Eraki M, Feilzer AJ (2007). The effect of direct and indirect water storage on the microtensile dentin bond strength of a total-etch and two self-etching adhesives. Am J Dent 20:370374.

3. Abdalla Al, El Zohairy AA, Aboushelib MM, Feilzer AJ (2007). Influence of thermal and mechanical load cycling on the microtensile bond strength of self-etching adhesives. Am J Dent 20:250-254.

4. Abdalla Al, Elsayed HY, Garcia-Godoy F (2008). Effect of hydrostatic pulpal water pressure on microtensile bond strength of self-etch adhesives to dentin. Am J Dent 21:233-238.

5. Abdalla Al, Feilzer AJ (2008). Four-year water degradation of a total-etch and two self-etching adhesives bonded to dentin. J Dent 36:611-617.

6. Abu-Hanna A, Gordan VV (2004). Evaluation of etching time on dentin bond strength using single bottle bonding systems. J Adhes Dent 6:105-110.

7. Adebayo OA, Burrow MF, Tyas MJ (2008). Dentine bonding after CPP-ACP paste treatment with and without conditioning. J Dent 36:1013-1024.

8. Aguilar-Mendoza JA, Rosales-Leal JI, Rodriguez-Valverde MA, Gonzalez-Lopez S, CabrerizoVilchez MA (2008). Wettability and bonding of self-etching dental adhesives. Influence of the smear layer. Dent Mater 24:994-1000.

9. Al-Ammar A, Drummond JL, Bedran-Russo AK (2009). The use of collagen cross-linking agents to enhance dentin bond strength. J Biomed Mater Res B Appl Biomater 91:419-424.

10. Al-Nahedh $\mathrm{H}$, Ateyah $\mathrm{N}$ (2006). Effect of different bonding conditions on the shear bond strength of two compomers to bovine dentin. J Contemp Dent Pract 7:9-16.

11. Albuquerque M, Pegoraro M, Mattei G, Reis A, Loguercio AD (2008). Effect of double-application or the application of a hydrophobic layer for improved efficacy of one-step self-etch systems in enamel and dentin. Oper Dent 33:564-570.

12. Ansari ZJ, Sadr A, Moezizadeh M, Aminian R, Ghasemi A, Shimada Y, Tagami J, Ansari SJ, Moayedi S (2008). Effects of one-year storage in water on bond strength of self-etching adhesives to enamel and dentin. Dent Mater J 27:266-272.

13. Ari H, Donmez N, Belli S (2008). Effect of Artificial Saliva Contamination on Bond Strength to Pulp Chamber Dentin. Eur J Dent 2:86-90.

14. Arias VG, Bedran-de-Castro AK, Pimenta LA (2005). Effects of sodium hypochlorite gel and sodium hypochlorite solution on dentin bond strength. J Biomed Mater Res B Appl Biomater 72:339-344.

15. Arrais CA, Giannini M, Nakajima M, Tagami J (2004). Effects of additional and extended acid etching on bonding to caries-affected dentine. Eur J Oral Sci 112:458-464.

16. Asaka Y, Amano S, Rikuta A, Kurokawa H, Miyazaki M, Platt JA, Moore BK (2007). Influence of thermal cycling on dentin bond strengths of single-step self-etch adhesive systems. Oper Dent 32:73-78.

17. Asaka Y, Miyazaki M, Takamizawa T, Tsubota K, Moore BK (2006). Influence of delayed placement of composites over cured adhesives on dentin bond strength of single-application self-etch systems. Oper Dent 31:18-24.

18. Asaka Y, Yamaguchi K, Inage H, Takamizawa T, Kurokawa H, Rikuta A, Kuroda T, Miyazaki M (2006). Effect of thermal cycling on bond strengths of single-step self-etch adhesives to bovine dentin. J Oral Sci 48:63-69.

19. Asmussen E, Peutzfeldt A (2005). Resin composites: strength of the bond to dentin versus surface energy parameters. Dent Mater 21:1039-1043.

20. Atash R, Van den Abbeele A (2005). Bond strengths of eight contemporary adhesives to enamel and to dentine: an in vitro study on bovine primary teeth. Int J Paediatr Dent 15:264-273.

21. Bagis B, Turkarslan S, Tezvergil-Mutluay A, Uctasli S, Vallittu PK, Lassila LV (2008). Effect of ultrasonic agitation on bond strength of self-etching adhesives to dentin. J Adhes Dent 10:441-445.

22. Banomyong D, Palamara JE, Burrow MF, Messer HH (2007). Effect of dentin conditioning on dentin permeability and micro-shear bond strength. Eur J Oral Sci 115:502-509.

23. Barbosa de Souza F, Silva CH, Guenka Palma Dibb R, Sincler Delfino C, Carneiro de Souza Beatrice $L$ (2005). Bonding performance of different adhesive systems to deproteinized dentin: microtensile bond strength and scanning electron microscopy. J Biomed Mater Res B Appl Biomater 75:158-167.

24. Belli S, Ozcopur B, Yesilyurt C, Bulut G, Ding X, Dorsman G (2009). The effect of loading on muTBS of four all-in-one adhesives on bonding to dentin. J Biomed Mater Res B Appl Biomater 91:948-956.

25. Betamar N, Cardew G, Van Noort R (2007). Influence of specimen designs on the microtensile bond strength to dentin. J Adhes Dent 9:159-168.

26. Borges GA, Spohr AM, de Oliveira WJ, Correr-Sobrinho L, Correr AB, Borges LH (2006). Effect of refrigeration on bond strength of self-etching adhesive systems. Braz Dent J 17:186-190.

27. Bortolotto T, Onisor I, Krejci I, Ferrari M, Tay FR, Bouillaguet S (2008). Effect of cyclic loading under enzymatic activity on resin-dentin interfaces of two self-etching adhesives. Dent Mater 24:178-184.

Published version: $\underline{\text { http://dx.doi.org/10.1177/0022034511431251 }}$

Journal homepage: http://jdr.sagepub.com/ 
28. Botta SB, da Ana PA, Zezell DM, Powers JM, Matos AB (2007). Adhesion after erbium, chromium:yttrium-scandium-gallium-garnet laser application at three different irradiation conditions. Lasers Med Sci 24:67-73.

29. Brackett WW, Ito S, Tay FR, Haisch LD, Pashley DH (2005). Microtensile dentin bond strength of self-etching resins: effect of a hydrophobic layer. Oper Dent 30:733-738.

30. Brackett WW, Tay FR, Looney SW, Ito S, Haisch LD, Pashley DH (2008). The effect of subject age on the microtensile bond strengths of a resin and a resin-modified glass ionomer adhesive to tooth structure. Oper Dent 33:282-286.

31. Brackett WW, Tay FR, Looney SW, Ito S, Haisch LD, Pashley DH (2008). Microtensile dentin and enamel bond strengths of recent self-etching resins. Oper Dent 33:89-95.

32. Bradna P, Vrbova R, Dudek M, Roubickova A, Housova D (2008). Comparison of bonding performance of self-etching and etch-and-rinse adhesives on human dentin using reliability analysis. J Adhes Dent 10:423-429.

33. Breschi L, Cammelli F, Visintini E, Mazzoni A, Vita F, Carrilho M, Cadenaro M, Foulger S, Mazzoti G, Tay FR, Di Lenarda R, Pashley D (2009). Influence of chlorhexidine concentration on the durability of etch-and-rinse dentin bonds: a 12-month in vitro study. J Adhes Dent 11:191-198.

34. Breschi L, Mazzoni A, Pashley DH, Pasquantonio G, Ruggeri A, Suppa P, Mazzotti G, Di Lenarda R, Tay FR (2006). Electric-current-assisted application of self-etch adhesives to dentin. J Dent Res 85:1092-1096.

35. Brulat N, Rocca JP, Leforestier E, Fiorucci G, Nammour S, Bertrand MF (2009). Shear bond strength of self-etching adhesive systems to Er:YAG-laser-prepared dentin. Lasers Med Sci 24:5357.

36. Bulucu B, Yesilyurt C, Cakir S, Meydan AD (2006). Influence of radiation on bond strength. J Adhes Dent 8:217-221.

37. Burrow MF, Harada N, Kitasako Y, Nikaido T, Tagami J (2005). Seven-year dentin bond strengths of a total- and self-etch system. Eur J Oral Sci 113:265-270.

38. Burrow MF, Kitasako Y, Thomas CD, Tagami J (2008). Comparison of enamel and dentin microshear bond strengths of a two-step self-etching priming system with five all-in-one systems. Oper Dent 33:456-460.

39. Cadenaro M, Delise C, Antoniollo F, Navarra OC, Di Lenarda R, Breschi L (2009). Enamel and dentin bond strength following gaseous ozone application. J Adhes Dent 11:287-292.

40. Camargo MA, Roda MI, Marques MM, de Cara AA (2008). Micro-tensile bond strength to bovine sclerotic dentine: influence of surface treatment. J Dent 36:922-927.

41. Campos EA, Correr GM, Leonardi DP, Barato-Filho F, Gonzaga CC, Zielak JC (2009). Chlorhexidine diminishes the loss of bond strength over time under simulated pulpal pressure and thermo-mechanical stressing. J Dent 37:108-114.

42. Can-Karabulut DC, Oz FT, Karabulut B, Batmaz I, Ilk O (2009). Adhesion to primary and permanent dentin and a simple model approach. Eur J Dent 3:32-41.

43. Cardoso MV, Coutinho E, Ermis RB, Poitevin A, Van Landuyt K, De Munck J, Carvalho RC, Lambrechts P, Van Meerbeek B (2008). Influence of Er,Cr:YSGG laser treatment on the microtensile bond strength of adhesives to dentin. J Adhes Dent 10:25-33.

44. Cardoso MV, Coutinho E, Ermis RB, Poitevin A, Van Landuyt K, De Munck J, Carvalho RC, Van Meerbeek B (2008). Influence of dentin cavity surface finishing on micro-tensile bond strength of adhesives. Dent Mater 24:492-501.

45. Cardoso MV, Moretto SG, de Carvalho RC, Russo EM (2008). Influence of intrapulpal pressure simulation on the bond strength of adhesive systems to dentin. Braz Oral Res 22:170-175.

46. Cardoso Pde C, Loguercio AD, Vieira LC, Baratieri LN, Reis A (2005). Effect of prolonged application times on resin-dentin bond strengths. J Adhes Dent 7:143-149.

47. Cardoso Pde C, Lopes GC, Vieira LC, Baratieri LN (2005). Effect of solvent type on microtensile bond strength of a total-etch one-bottle adhesive system to moist or dry dentin. Oper Dent 30:376381.

48. Carrilho MR, Carvalho RM, Tay FR, Yiu C, Pashley DH (2005). Durability of resin-dentin bonds related to water and oil storage. Am J Dent 18:315-319.

49. Carvalho CA, Cantoro A, Mazzoni A, Goracci C, Breschi L, Ferrari M (2009). Effect of ethanol application on post-luting to intraradicular dentine. Int Endod J 42:129-135.

50. Carvalho CN, de Oliveira Bauer JR, Loguercio AD, Reis A (2007). Effect of ZOE temporary restoration on resin-dentin bond strength using different adhesive strategies. J Esthet Restor Dent 19:144-52; discussion 153.

51. Casagrande L, de Hipolito V, de Goes MF, Barata JS, Garcia-Godoy F, de Araujo FB (2006). Bond strength and failure patterns of adhesive restorations in primary teeth aged in the oral environment. Am J Dent 19:279-282.

52. Cassoni A, Youssef MN, Prokopowitsch I (2005). Bond strength of a dentin bonding system using two techniques of polymerization: visible-light and argon laser. Photomed Laser Surg 23:493-497.

53. Cavalcante LM, Erhardt MC, Bedran-de-Castro AK, Pimenta LA, Ambrosano GM (2006). Influence of different tests used to measure the bond strength to dentin of two adhesive systems. Am J Dent 19:37-40. 
54. Cavalcanti AN, Mitsui FH, Ambrosano GM, Marchi GM (2007). Influence of adhesive systems and flowable composite lining on bond strength of class II restorations submitted to thermal and mechanical stresses. J Biomed Mater Res B Appl Biomater 80:52-58.

55. Cekic-Nagas I, Ergun G, Tezvergil A, Vallittu PK, Lassila LV (2008). Effect of fiber-reinforced composite at the interface on bonding of resin core system to dentin. Dent Mater J 27:736-743.

56. Cekic-Nagas I, Ergun G, Vallittu PK, Lassila LV (2008). Influence of polymerization mode on degree of conversion and micropush-out bond strength of resin core systems using different adhesive systems. Dent Mater J 27:376-385.

57. Celik C, Ozel Y, Bagis B, Erkut S (2010). Effect of Laser Irradiation and Cavity Disinfectant Application on the Microtensile Bond Strength of Different Adhesive Systems. Photomed Laser Surg 28:267-272.

58. Celik EU, Ergucu Z, Turkun LS, Turkun M (2006). Shear bond strength of different adhesives to Er:YAG laser-prepared dentin. J Adhes Dent 8:319-325.

59. Chen LJ, Meng QF, Chen YM, Smales RJ, Yip KH (2008). Effect of fluoride iontophoresis on the microtensile bond strength between dentin and two adhesive systems. J Dent 36:697-702.

60. Chiba Y, Rikuta A, Yasuda G, Yamamoto A, Takamizawa T, Kurokawa H, Ando S, Miyazaki M (2006). Influence of moisture conditions on dentin bond strength of single-step self-etch adhesive systems. J Oral Sci 48:131-137.

61. Chiba Y, Yamaguchi K, Miyazaki M, Tsubota K, Takamizawa T, Moore BK (2006). Effect of airdrying time of single-application self-etch adhesives on dentin bond strength. Oper Dent 31:233239.

62. Coelho PG, Calamia C, Harsono M, Thompson VP, Silva NR (2008). Laboratory and FEA evaluation of dentin-to-composite bonding as a function adhesive layer thickness. Dent Mater 24:1297-1303.

63. Colucci V, Lucisano MP, do Amaral FL, Pecora JD, Palma-Dibb RG, Corona SA (2008). Influence of water flow rate on shear bond strength of resin composite to Er:YAG cavity preparation. Am J Dent 21:124-128.

64. Courson F, Bouter D, Ruse ND, Degrange M (2005). Bond strengths of nine current dentine adhesive systems to primary and permanent teeth. J Oral Rehabil 32:296-303.

65. D'Arcangelo C, Vanini L, Prosperi GD, Di Bussolo G, De Angelis F, D'Amario M, Caputi S (2009). The influence of adhesive thickness on the microtensile bond strength of three adhesive systems. $J$ Adhes Dent 11:109-115.

66. Dal-Bianco K, Pellizzaro A, Patzlaft R, de Oliveira Bauer JR, Loguercio AD, Reis A (2006). Effects of moisture degree and rubbing action on the immediate resin-dentin bond strength. Dent Mater 22:1150-1156.

67. Dantas DC, Ribeiro Al, Lima LH, de Lima MG, Guenes GM, Braz AK, Braz R (2008). Influence of water storage time on the bond strength of etch-and-rinse and self-etching adhesive systems. Braz Dent J 19:219-223.

68. De Goes MF, Giannini M, Di Hipolito V, Carrilho MR, Daronch M, Rueggeberg FA (2008). Microtensile bond strength of adhesive systems to dentin with or without application of an intermediate flowable resin layer. Braz Dent J 19:51-56.

69. De Goes MF, Giannini M, Foxton RM, Nikaido T, Tagami J (2007). Microtensile bond strength between crown and root dentin and two adhesive systems. J Prosthet Dent 97:223-228.

70. De Munck J, Arita A, Shirai K, Van Landuyt KL, Coutinho E, Poitevin A, Peumans M, Lambrechts P. Van Meerbeek B (2007). Microrotary fatigue resistance of a HEMA-free all-in-one adhesive bonded to dentin. J Adhes Dent 9:373-379.

71. De Munck J, Braem M, Wevers M, Yoshida Y, Inoue S, Suzuki K, Lambrechts P, Van Meerbeek B (2005). Micro-rotary fatigue of tooth-biomaterial interfaces. Biomaterials 26:1145-1153.

72. De Munck J, Shirai K, Yoshida Y, Inoue S, Van Landuyt K, Lambrechts P, Suzuki K, Shintani H, Van Meerbeek B (2006). Effect of water storage on the bonding effectiveness of 6 adhesives to Class I cavity dentin. Oper Dent 31:456-465.

73. De Munck J, Van Landuyt K, Coutinho E, Poitevin A, Peumans M, Lambrechts P, Van Meerbeek B (2005). Micro-tensile bond strength of adhesives bonded to Class-I cavity-bottom dentin after thermo-cycling. Dent Mater 21:999-1007.

74. De Munck J, Van Landuyt KL, Coutinho E, Poitevin A, Peumans M, Lambrechts P, Braem M, Van Meerbeek B (2005). Fatigue resistance of dentin/composite interfaces with an additional intermediate elastic layer. Eur J Oral Sci 113:77-82.

75. De Munck J, Vargas M, Iracki J, Van Landuyt K, Poitevin A, Lambrechts P, Van Meerbeek B (2005) One-day bonding effectiveness of new self-etch adhesives to bur-cut enamel and dentin. Oper Dent 30:39-49.

76. de Silva AL, Lima DA, de Souza GM, dos Santos CT, Paulillo LA (2006). Influence of additional adhesive application on the microtensile bond strength of adhesive systems. Oper Dent 31:562-568.

77. Dias WR, Pereira PN, Swift EJ Jr (2004). Effect of bur type on microtensile bond strengths of selfetching systems to human dentin. J Adhes Dent 6:195-203.

78. Ding PG, Wolff D, Pioch T, Staehle HJ, Dannewitz B (2009). Relationship between microtensile bond strength and nanoleakage at the composite-dentin interface. Dent Mater 25:135-141.

79. do Amaral RC, Stanislawczuk R, Zander-Grande C, Michel MD, Reis A, Loguercio AD (2009). Active application improves the bonding performance of self-etch adhesives to dentin. J Dent 37:82-90.

Published version: $\underline{\text { http://dx.doi.org/10.1177/0022034511431251 }}$

Journal homepage: http://jdr.sagepub.com/ 
80. do Amaral RC, Stanislawczuk R, Zander-Grande C, Michel MD, Reis A, Loguercio AD (2009). Active application improves the bonding performance of self-etch adhesives to dentin. J Dent 37:82-90.

81. Doi J, Itota T, Torii Y, Nakabo S, Yoshiyama M (2004). Micro-tensile bond strength of self-etching primer adhesive systems to human coronal carious dentin. J Oral Rehabil 31:1023-1028.

82. Donmez N, Belli S, Pashley DH, Tay FR (2005). Ultrastructural correlates of in vivo/in vitro bond degradation in self-etch adhesives. J Dent Res 84:355-359.

83. Dos Santos PA, Garcia PP, Palma-Dibb RG (2005). Shear bond strength of adhesive systems to enamel and dentin. Thermocycling influence. J Mater Sci Mater Med 16:727-732.

84. dos Santos PH, Sinhoreti MA, Consani S, Sobrinho LC, Adabo GL, Vaz LG (2005). Effect of cyclic compressive loading on the bond strength of an adhesive system to dentin after collagen removal. $J$ Adhes Dent 7:127-131.

85. Duarte S Jr, Phark JH, Varjao FM, Sadan A (2009). Nanoleakage, ultramorphological characteristics, and microtensile bond strengths of a new low-shrinkage composite to dentin after artificial aging. Dent Mater 25:589-600.

86. Eckert GJ, Platt JA (2007). A statistical evaluation of microtensile bond strength methodology for dental adhesives. Dent Mater 23:385-391.

87. El-Araby AM, Talic YF (2007). The effect of thermocycling on the adhesion of self-etching adhesives on dental enamel and dentin. J Contemp Dent Pract 8:17-24.

88. El-Kholany NR, Abdelaziz KM, Zaghloul NM, Aboulenien N (2005). Bonding of single-component adhesives to dentin following chemomechanical caries removal. J Adhes Dent 7:281-287.

89. Ergucu Z, Celik EU, Unlu N, Turkun M, Ozer F (2009). Effect of Er,Cr:YSGG laser on the microtensile bond strength of two different adhesives to the sound and caries-affected dentin. Oper Dent 34:460-466.

90. Erhardt MC, Osorio E, Aguilera FS, Proenca JP, Osorio R, Toledano M (2008). Influence of dentin acid-etching and $\mathrm{NaOCl}$-treatment on bond strengths of self-etch adhesives. Am J Dent 21:44-48.

91. Erhardt MC, Osorio R, Toledano M (2008). Dentin treatment with MMPs inhibitors does not alter bond strengths to caries-affected dentin. J Dent 36:1068-1073.

92. Erhardt MC, Rodrigues JA, Valentino TA, Ritter AV, Pimenta LA (2008). In vitro microTBS of onebottle adhesive systems: sound versus artificially-created caries-affected dentin. J Biomed Mater Res B Appl Biomater 86:181-187.

93. Erhardt MC, Toledano M, Osorio R, Pimenta LA (2008). Histomorphologic characterization and bond strength evaluation of caries-affected dentin/resin interfaces: effects of long-term water exposure. Dent Mater 24:786-798.

94. Ermis RB, De Munck J, Cardoso MV, Coutinho E, Van Landuyt KL, Poitevin A, Lambrechts P, Van Meerbeek B (2008). Bond strength of self-etch adhesives to dentin prepared with three different diamond burs. Dent Mater 24:978-985.

95. F e Silva AL, Pereira GD, Dias CT, Sartini Paulillo LA (2006). Effect of the composite photoactivation mode on microtensile bond strength and Knoop microhardness. Dent Mater 22:203-210.

96. Fawzy AS, Amer MA, El-Askary FS (2008). Sodium hypochlorite as dentin pretreatment for etchand-rinse single-bottle and two-step self-etching adhesives: atomic force microscope and tensile bond strength evaluation. J Adhes Dent 10:135-144.

97. Felix SA, Gonzalez-Lopez S, Mauricio PD, Aguilar-Mendoza JA, Bolanos-Carmona MV (2007). Effects of filling techniques on the regional bond strength to lateral walls in Class I cavities. Oper Dent 32:602-609.

98. Ferreira LS, Ferreira LS, Francci C, Navarro RS, Calheiros FC, Eduardo Cde P (2009). Effects of $\mathrm{Nd}$ :YAG laser irradiation on the hybrid layer of different adhesive systems. J Adhes Dent 11:117125.

99. Finger WJ, Shao B, Hoffmann M, Kanehira M, Endo T, Komatsu M (2007). Does application of phase-separated self-etching adhesives affect bond strength? J Adhes Dent 9:169-173.

100. Finger WJ, Tani C (2005). Effect of application mode on bonding performance of self-etching adhesives. Am J Dent 18:41-44.

101. Foxton RM, Melo L, Stone DG, Pilecki P, Sherriff M, Watson TF (2008). Long-term durability of onestep adhesive-composite systems to enamel and dentin. Oper Dent 33:651-657.

102. Franca FM, dos Santos AJ, Lovadino JR (2007). Influence of air abrasion and long-term storage on the bond strength of self-etching adhesives to dentin. Oper Dent 32:217-224.

103. Frankenberger R, Lohbauer U, Tay FR, Taschner M, Nikolaenko SA (2007). The effect of different air-polishing powders on dentin bonding. J Adhes Dent 9:381-389.

104. Frankenberger R, Pashley DH, Reich SM, Lohbauer U, Petschelt A, Tay FR (2005). Characterisation of resin-dentine interfaces by compressive cyclic loading. Biomaterials 26:20432052.

105. Frankenberger R, Strobel WO, Lohbauer U, Kramer N, Petschelt A (2004). The effect of six years of water storage on resin composite bonding to human dentin. J Biomed Mater Res B Appl Biomater 69:25-32.

106. Furuse AY, Peutzfeldt A, Asmussen E (2008). Effect of evaporation of solvents from one-step, selfetching adhesives. J Adhes Dent 10:35-39.

107. Gamborgi GP, Loguercio AD, Reis A (2007). Influence of enamel border and regional variability on durability of resin-dentin bonds. J Dent 35:371-376. 
108. Garcia FC, Almeida JC, Osorio R, Carvalho RM, Toledano M (2009). Influence of drying time and temperature on bond strength of contemporary adhesives to dentine. J Dent 37:315-320.

109. Garcia RN, de Goes MF, Giannini M (2007). Effect of water storage on bond strength of self-etching adhesives to dentin. J Contemp Dent Pract 8:46-53.

110. Gernhardt CR, Bekes K, Hahn P, Schaller HG (2008). Influence of pressure application before lightcuring on the bond strength of adhesive systems to dentin. Braz Dent J 19:62-67.

111. Gernhardt CR, Schaller HG, Kielbassa AM (2005). The influence of human plasma used for dentin perfusion on tensile bond strength of different light-curing materials. Am J Dent 18:318-322.

112. Goncalves M, Corona SA, Borsatto MC, Pecora JD, Dibb RG (2005). Influence of pulse frequency Er:YAG laser on the tensile bond strength of a composite to dentin. Am J Dent 18:165-167.

113. Gurgan S, Kiremitci A, Cakir FY, Gorucu J, Alpaslan T, Yazici E, Gutknecht N (2008). Shear bond strength of composite bonded to Er,Cr:YSGG laser-prepared dentin. Photomed Laser Surg 26:495500.

114. Gurgan S, Kiremitci A, Cakir FY, Yazici E, Gorucu J, Gutknecht N (2007). Shear bond strength of composite bonded to erbium:yttrium-aluminum-garnet laser-prepared dentin. Lasers Med Sci 24:117-112.

115. Harnirattisai C, Senawongse P, Tagami J (2007). Microtensile bond strengths of two adhesive resins to discolored dentin after amalgam removal. J Dent Res 86:232-236.

116. Hashimoto M, Fujita S, Kaga M, Yawaka Y (2007). In vitro durability of one-bottle resin adhesives bonded to dentin. Dent Mater J 26:677-686.

117. Hashimoto M, Fujita S, Kaga M, Yawaka Y (2008). Effect of water on bonding of one-bottle selfetching adhesives. Dent Mater J 27:172-178.

118. Hashimoto M, Sano H, Yoshida E, Hori M, Kaga M, Oguchi H, Pashley DH (2004). Effects of multiple adhesive coatings on dentin bonding. Oper Dent 29:416-423.

119. Hashimoto M, Tay FR, Svizero NR, de Gee AJ, Feilzer AJ, Sano H, Kaga M, Pashley DH (2006). The effects of common errors on sealing ability of total-etch adhesives. Dent Mater 22:560-568.

120. He Z, Shimada Y, Sadr A, Ikeda M, Tagami J (2008). The effects of cavity size and filling method on the bonding to Class I cavities. J Adhes Dent 10:447-453.

121. Hebling J, Castro FL, Costa CA (2007). Adhesive performance of dentin bonding agents applied in vivo and in vitro. Effect of intrapulpal pressure and dentin depth. J Biomed Mater Res B Appl Biomater 83:295-303.

122. Helvatjoglu-Antoniades M, Koliniotou-Kubia E, Dionyssopoulos P (2004). The effect of thermal cycling on the bovine dentine shear bond strength of current adhesive systems. J Oral Rehabil 31:911-917.

123. Hosaka K, Nakajima M, Monticelli F, Carrilho M, Yamauti M, Aksornmuang J, Nishitani Y, Tay FR, Pashley DH, Tagami J (2007). Influence of hydrostatic pulpal pressure on the microtensile bond strength of all-in-one self-etching adhesives. J Adhes Dent 9:437-442.

124. Hosaka K, Nakajima M, Yamauti M, Aksornmuang J, Ikeda M, Foxton RM, Pashley DH, Tagami J (2007). Effect of simulated pulpal pressure on all-in-one adhesive bond strengths to dentine. J Dent 35:207-213.

125. Hosoya Y, Kawada E, Ushigome T, Oda Y, Garcia-Godoy F (2006). Micro-tensile bond strength of sound and caries-affected primary tooth dentin measured with original designed jig. J Biomed Mater Res B Appl Biomater 77:241-248.

126. Hosoya Y, Shinkawa H, Suefiji C, Nozaka K, Garcia-Godoy F (2004). Effects of diamond bur particle size on dentin bond strength. Am J Dent 17:359-364.

127. Huang MS, Li MT, Huang FM, Ding SJ (2004). The effect of thermocycling and dentine pretreatment on the durability of the bond between composite resin and dentine. J Oral Rehabil 31:492499.

128. lida Y, Nikaido T, Kitayama S, Takagaki T, Inoue G, Ikeda M, M Foxton R, Tagami J (2009). Evaluation of dentin bonding performance and acid-base resistance of the interface of two-step selfetching adhesive systems. Dent Mater J 28:493-500.

129. Ikeda M, Tsubota K, Takamizawa T, Yoshida T, Miyazaki M, Platt JA (2008). Bonding durability of single-step adhesives to previously acid-etched dentin. Oper Dent 33:702-709.

130. Inoue S, Koshiro K, Yoshida Y, De Munck J, Nagakane K, Suzuki K, Sano H, Van Meerbeek B (2005). Hydrolytic stability of self-etch adhesives bonded to dentin. J Dent Res 84:1160-1164.

131. Irie M, Hatanaka K, Suzuki K, Watts DC (2006). Immediate versus water-storage performance of Class $\vee$ flowable composite restoratives. Dent Mater 22:875-883.

132. Irie M, Suzuki K, Watts DC (2004). Immediate performance of self-etching versus system adhesives with multiple light-activated restoratives. Dent Mater 20:873-880.

133. Ishii T, Ohara N, Oshima A, Koizumi H, Nakazawa M, Masuno T, Matsumura H (2008). Bond strength to bovine dentin of a composite core build-up material combined with four different bonding agents. J Oral Sci 50:329-333.

134. Ishikawa A, Shimada Y, Foxton RM, Tagami J (2007). Micro-tensile and micro-shear bond strengths of current self-etch adhesives to enamel and dentin. Am J Dent 20:161-166.

135. Ito S, Tay FR, Hashimoto M, Yoshiyama M, Saito T, Brackett WW, Waller JL, Pashley DH (2005). Effects of multiple coatings of two all-in-one adhesives on dentin bonding. J Adhes Dent 7:133-141. 
136. Jacobsen T, Finger WJ, Kanehira M (2006). Air-drying time of self-etching adhesives vs bonding efficacy. J Adhes Dent 8:387-392.

137. Jacques $P$, Hebling J (2005). Effect of dentin conditioners on the microtensile bond strength of a conventional and a self-etching primer adhesive system. Dent Mater 21:103-109.

138. Kameyama A, Aizawa K, Kato J, Hirai Y (2009). Tensile Bond Strength of Single-Step Self-Etch Adhesives to Er:YAG Laser-Irradiated Dentin. Photomed Laser Surg 27:3-10.

139. Kaneshiro AV, Imazato S, Ebisu S (2007). Comparison of bonding ability of single-step self-etching adhesives with different etching aggressiveness to root dentin. Dent Mater J 26:773-784.

140. Karakaya S, Unlu N, Say EC, Ozer F, Soyman M, Tagami J (2008). Bond strengths of three different dentin adhesive systems to sclerotic dentin. Dent Mater J 27:471-479.

141. Kenshima S, Reis A, Uceda-Gomez N, Tancredo Lde L, Filho LE, Nogueira FN, Loguercio AD (2005). Effect of smear layer thickness and $\mathrm{pH}$ of self-etching adhesive systems on the bond strength and gap formation to dentin. J Adhes Dent 7:117-126.

142. Kim JS, Choi YH, Cho BH, Son HH, Lee IB, Um CM, Kim CK (2006). Effect of light-cure time of adhesive resin on the thickness of the oxygen-inhibited layer and the microtensile bond strength to dentin. J Biomed Mater Res B Appl Biomater 78:115-123.

143. Kim SY, Lee IB, Cho BH, Son $\mathrm{HH}$, Um CM (2006). Curing effectiveness of a light emitting diode on dentin bonding agents. J Biomed Mater Res B Appl Biomater 77:164-170.

144. Kimmes NS, Olson TL, Shaddy RS, Latta MA (2006). Effect of ViscoStat and ViscoStat Plus on composite shear bond strength in the presence and absence of blood. J Adhes Dent 8:363-366.

145. King NM, Tay FR, Pashley DH, Hashimoto M, Ito S, Brackett WW, Garcia-Godoy F, Sunico M (2005). Conversion of one-step to two-step self-etch adhesives for improved efficacy and extended application. Am J Dent 18:126-134.

146. Klein-Junior CA, Zander-Grande C, Amaral R, Stanislawczuk R, Garcia EJ, Baumhardt-Neto R, Meier MM, Loguercio AD, Reis A (2008). Evaporating solvents with a warm air-stream: effects on adhesive layer properties and resin-dentin bond strengths. J Dent 36:618-625.

147. Knobloch LA, Gailey D, Azer S, Johnston WM, Clelland N, Kerby RE (2007). Bond strengths of oneand two-step self-etch adhesive systems. J Prosthet Dent 97:216-222.

148. Koase K, Inoue S, Noda M, Tanaka T, Kawamoto C, Takahashi A, Nakaoki Y, Sano H (2004). Effect of bur-cut dentin on bond strength using two all-in-one and one two-step adhesive systems. J Adhes Dent 6:97-104.

149. Komori PC, Pashley DH, Tjaderhane L, Breschi L, Mazzoni A, de Goes MF, Wang L, Carrilho MR (2009). Effect of $2 \%$ chlorhexidine digluconate on the bond strength to normal versus caries-affected dentin. Oper Dent 34:157-165.

150. Korkmaz Y, Attar N (2007). Dentin bond strength of composites with self-etching adhesives using LED curing lights. J Contemp Dent Pract 8:34-42.

151. Korkmaz Y, Baseren M (2008). Effect of antibacterial varnishes applied to root dentin on shear bond strength of tooth-colored restorative materials. Oper Dent 33:65-71.

152. Koshiro K, Inoue S, Niimi K, Koase K, Sano H (2005). Bond strength and SEM observation of CO2 laser irradiated dentin, bonded with simplified-step adhesives. Oper Dent 30:170-179.

153. Koyuturk AE, Sengun A, Ozer F, Sener Y, Gokalp A (2006). Shear bond strengths of self-etching adhesives to caries-affected dentin on the gingival wall. Dent Mater J 25:59-65.

154. Kuphasuk W, Harnirattisai C, Senawongse P, Tagami J (2007). Bond strengths of two adhesive systems to dentin contaminated with a hemostatic agent. Oper Dent 32:399-405.

155. Kurokawa R, Finger WJ, Hoffmann M, Endo T, Kanehira M, Komatsu M, Manabe A (2007). Interactions of self-etch adhesives with resin composites. J Dent 35:923-929.

156. Lattaa MA (2007). Shear bond strength and physicochemical interactions of XP Bond. J Adhes Dent 9 Suppl 2:245-248.

157. Lee BS, Lin PY, Chen MH, Hsieh TT, Lin CP, Lai JY, Lan WH (2007). Tensile bond strength of Er,Cr:YSGG laser-irradiated human dentin and analysis of dentin-resin interface. Dent Mater 23:570-578.

158. Lee JJ, Nettey-Marbell A, Cook A Jr, Pimenta LA, Leonard R, Ritter AV (2007). Using extracted teeth for research: the effect of storage medium and sterilization on dentin bond strengths. $\mathrm{J} A m$ Dent Assoc 138:1599-1603.

159. Lodovici E, Reis A, Geraldeli S, Ferracane JL, Ballester RY, Rodrigues Filho LE (2009). Does adhesive thickness affect resin-dentin bond strength after thermal/load cycling? Oper Dent 34:5864.

160. Loguercio AD, Barroso LP, Grande RH, Reis A (2005). Comparison of intra- and intertooth resindentin bond strength variability. J Adhes Dent 7:151-158.

161. Loguercio AD, Loeblein F, Cherobin T, Ogliari F, Piva E, Reis A (2009). Effect of solvent removal on adhesive properties of simplified etch-and-rinse systems and on bond strengths to dry and wet dentin. J Adhes Dent 11:213-219.

162. Loguercio AD, Uceda-Gomez N, Carrilho MR, Reis A (2005). Influence of specimen size and regional variation on long-term resin-dentin bond strength. Dent Mater 21:224-231.

163. Lopes GC, Cardoso PC, Vieira LC, Baratieri LN, Rampinelli K, Costa G (2006). Shear bond strength of acetone-based one-bottle adhesive systems. Braz Dent J 17:39-43. 
164. Lopes GC, Perdigao J, Lopes Mde F, Vieira LC, Baratieri LN, Monteiro S Jr (2006). Dentin bond strengths of simplified adhesives: effect of dentin depth. Compend Contin Educ Dent 27:340-345.

165. Ma S, Nakajima KF, Nishiyama N (2009). Effects of storage temperature on the shelf life of one-step and two-step self-etch adhesives. Oper Dent 34:472-480.

166. Maggio VB, Vandewalle KS (2009). Effect of a simulated high-heat environment on bond strengths of dental adhesive systems. Mil Med 174:193-196.

167. Magne P, So WS, Cascione D (2007). Immediate dentin sealing supports delayed restoration placement. J Prosthet Dent 98:166-174.

168. Mallmann A, Melo RM, Estrela V, Pelogia F, Campos L, Bottino MA, Valandro LF (2007). Adhesives with different pHs: effect on the MTBS of chemically activated and light-activated composites to human dentin. J Appl Oral Sci 15:265-269.

169. Manso AP, Marquezini L Jr, Silva SM, Pashley DH, Tay FR, Carvalho RM (2008). Stability of wet versus dry bonding with different solvent-based adhesives. Dent Mater 24:476-482.

170. Matos AB, Oliveira DC, Vieira SN, Netto NG, Powers JM (2008). Influence of oil contamination on in vitro bond strength of bonding agents to dental substrates. Am J Dent 21:101-104.

171. Maurin JC, Lagneau C, Durand M, Lissac M, Seux D (2006). Tensile and shear bond strength evaluation of a total-etch three-step and two self-etching one-step dentin bonding systems. J Adhes Dent 8:27-30.

172. Mazzoni A, Pashley DH, Ruggeri A Jr, Vita F, Falconi M, Di Lenarda R, Breschi L (2008). Adhesion to chondroitinase ABC treated dentin. J Biomed Mater Res B Appl Biomater 86:228-236.

173. Mazzoni A, Visintini E, Vita F, Pasquantonio G, Saboia VP, Ruggeri A Jr, Di Lenarda R, Dorigo E, Breschi $L$ (2009). ElectroBond improves immediate dentin microtensile bond strength of two etchand-rinse adhesives. J Adhes Dent 11:27-33.

174. Miguez PA, Pereira PN, Foxton RM, Walter R, Nunes MF, Swift EJ Jr (2004). Effects of flowable resin on bond strength and gap formation in Class I restorations. Dent Mater 20:839-845.

175. Mine A, De Munck J, Cardoso MV, Van Landuyt KL, Poitevin A, Kuboki T, Yoshida Y, Suzuki K, Lambrechts P, Van Meerbeek B (2009). Bonding effectiveness of two contemporary self-etch adhesives to enamel and dentin. J Dent 37:872-883.

176. Mine A, De Munck J, Van Landuyt KL, Poitevin A, Kuboki T, Yoshida Y, Suzuki K, Lambrechts P, Van Meerbeek B (2008). Bonding effectiveness and interfacial characterization of a HEMA/TEGDMA-free three-step etch\&rinse adhesive. J Dent 36:767-773.

177. Miranda C, Prates LH, Vieira Rde S, Calvo MC (2006). Shear bond strength of different adhesive systems to primary dentin and enamel. J Clin Pediatr Dent 31:35-40.

178. Mitsui FH, Peris AR, Cavalcanti AN, Marchi GM, Pimenta LA (2006). Influence of thermal and mechanical load cycling on microtensile bond strengths of total and self-etching adhesive systems. Oper Dent 31:240-247.

179. Nakaoki Y, Sasakawa W, Horiuchi S, Nagano F, Ikeda T, Tanaka T, Inoue S, Uno S, Sano H, Sidhu SK (2005). Effect of double-application of all-in-one adhesives on dentin bonding. J Dent 33:765772.

180. Nakornchai S, Harnirattisai C, Surarit R, Thiradilok S (2005). Microtensile bond strength of a totaletching versus self-etching adhesive to caries-affected and intact dentin in primary teeth. J Am Dent Assoc 136:477-483.

181. Nam KY, Kim JB, Jang BC, Kwon TY, Kim KH (2007). Effects of dentin bonding agents on bonding durability of a flowable composite to dentin. Dent Mater J 26:224-231.

182. Naughton WT, Latta MA (2005). Bond strength of composite to dentin using self-etching adhesive systems. Quintessence Int 36:259-262.

183. Nayif MM, Nakajima M, Foxton RM, Tagami J (2008). Bond strength and ultimate tensile strength of resin composite filled into dentine cavity; effect of bulk and incremental filling technique. $J$ Dent 36:228-234.

184. Neelima L, Sathish ES, Kandaswamy D (2008). Evaluation of microtensile bond strength of totaletch, self-etch, and glass ionomer adhesive to human dentin: an in vitro study. Indian J Dent Res 19:129-133.

185. Nishiyama N, Fujita K, Ikemi T, Maeda T, Suzuki K, Nemoto K (2005). Efficacy of varying the NMEP concentrations in the NMGly-NMEP self-etching primer on the resin-tooth bonding. Biomaterials 26:2653-2661.

186. O'Donnell JN, Schumacher GE, Antonucci JM, Skrtic D (2008). Adhesion of amorphous calcium phosphate composites bonded to dentin: A study in failure modality. J Biomed Mater Res B Appl Biomater 90:238-244.

187. O'Keefe KL, Pinzon LM, Rivera B, Powers JM (2005). Bond strength of composite to astringentcontaminated dentin using self-etching adhesives. Am J Dent 18:168-172.

188. Omar H, El-Badrawy W, El-Mowafy O, Atta O, Saleem B (2007). Microtensile bond strength of resin composite bonded to caries-affected dentin with three adhesives. Oper Dent 32:24-30.

189. Osorio R, Erhardt MC, Pimenta LA, Osorio E, Toledano M (2005). EDTA treatment improves resindentin bonds' resistance to degradation. J Dent Res 84:736-740.

190. Osorio R, Pisani-Proenca J, Erhardt MC, Osorio E, Aguilera FS, Tay FR, Toledano M (2008). Resistance of ten contemporary adhesives to resin-dentine bond degradation. J Dent 36:163-169. 
191. Ozer F, Sengun A, Ozturk B, Say EC, Tagami J (2005). Effect of tooth age on microtensile bond strength of two fluoride-releasing bonding agents. J Adhes Dent 7:289-295.

192. Ozok AR, Wu MK, De Gee AJ, Wesselink PR (2004). Effect of dentin perfusion on the sealing ability and microtensile bond strengths of a total-etch versus an all-in-one adhesive. Dent Mater 20:479486.

193. Pangsrisomboon B, Harnirattisai C, Nilsri K, Burrow MF (2007). Microtensile bond strength of selfetching adhesive systems to differently prepared dentin. Am J Dent 20:259-262.

194. Paradella TC, Fava M (2007). Bond strength of adhesive systems to human tooth enamel. Braz Oral Res 21:4-9.

195. Park JW, Lee KC (2004). The influence of salivary contamination on shear bond strength of dentin adhesive systems. Oper Dent 29:437-442.

196. Pasquantonio G, Tay FR, Mazzoni A, Suppa P, Ruggeri A Jr, Falconi M, Di Lenarda R, Breschi L (2007). Electric device improves bonds of simplified etch-and-rinse adhesives. Dent Mater 23:513518.

197. Pazinatto FB, Atta MT (2008). Influence of differently oriented dentin surfaces and the regional variation of specimens on adhesive layer thickness and bond strength. J Esthet Restor Dent 20:11928.

198. Perdigao J, Gomes G, Gondo R, Fundingsland JW (2006). In vitro bonding performance of all-inone adhesives. Part I--microtensile bond strengths. J Adhes Dent 8:367-373.

199. Pereira PN, Nunes MF, Miguez PA, Swift EJ Jr (2006). Bond strengths of a 1-step self-etching system to caries-affected and normal dentin. Oper Dent 31:677-681.

200. Peris AR, Mitsui FH, Lobo MM, Bedran-russo AK, Marchi GM (2007). Adhesive systems and secondary caries formation: Assessment of dentin bond strength, caries lesions depth and fluoride release. Dent Mater 23:308-316.

201. Peutzfeldt A, Asmussen E (2006). Influence of eugenol-containing temporary cement on bonding of self-etching adhesives to dentin. J Adhes Dent 8:31-34.

202. Piva E, Ogliari FA, Moraes RR, Cora F, Henn S, Correr-Sobrinho L (2008). Papain-based gel for biochemical caries removal: influence on microtensile bond strength to dentin. Braz Oral Res 22:364-370.

203. Poitevin A, De Munck J, Van Landuyt K, Coutinho E, Peumans M, Lambrechts P, Van Meerbeek B (2008). Critical analysis of the influence of different parameters on the microtensile bond strength of adhesives to dentin. J Adhes Dent 10:7-16.

204. Poitevin A, De Munck J, Van Landuyt K, Coutinho E, Peumans M, Lambrechts P, Van Meerbeek B (2007). Influence of three specimen fixation modes on the micro-tensile bond strength of adhesives to dentin. Dent Mater J 26:694-699.

205. Pongprueksa P, Kuphasuk W, Senawongse P (2007). Effect of elastic cavity wall and occlusal loading on microleakage and dentin bond strength. Oper Dent 32:466-475.

206. Prabhakar AR, Bedi S (2008). Effect of glutaraldehyde and ferric sulfate on shear bond strength of adhesives to primary dentin. J Indian Soc Pedod Prev Dent 26 Suppl 3:S109-S113.

207. Proenca JP, Polido M, Osorio E, Erhardt MC, Aguilera FS, Garcia-Godoy F, Osorio R, Toledano M (2007). Dentin regional bond strength of self-etch and total-etch adhesive systems. Dent Mater 23:1542-1548.

208. Purk JH, Ganne S, Parikh R, Dusevich V, Glaros A, Eick JD (2004). An in-vitro microtensile test of Scotchbond Multi-Purpose adhesive applied at different priming times. Oper Dent 29:693-697.

209. Purk JH, Healy M, Dusevich V, Glaros A, Eick JD (2006). In vitro microtensile bond strength of four adhesives tested at the gingival and pulpal walls of Class II restorations. J Am Dent Assoc 137:1414-1418.

210. Ramos AC, Esteves-Oliveira M, Arana-Chavez VE, de Paula Eduardo C (2010). Adhesives bonded to erbium:yttrium-aluminum-garnet laser-irradiated dentin: transmission electron microscopy, scanning electron microscopy and tensile bond strength analyses. Lasers Med Sci 25:181-189.

211. Reis A, Albuquerque M, Pegoraro M, Mattei G, Bauer JR, Grande RH, Klein-Junior CA, BaumhardtNeto R, Loguercio AD (2008). Can the durability of one-step self-etch adhesives be improved by double application or by an extra layer of hydrophobic resin? J Dent 36:309-315.

212. Reis A, de Carvalho Cardoso P, Vieira LC, Baratieri LN, Grande RH, Loguercio AD (2008). Effect of prolonged application times on the durability of resin-dentin bonds. Dent Mater 24:639-644.

213. Reis A, Grande RH, Oliveira GM, Lopes GC, Loguercio AD (2007). A 2-year evaluation of moisture on microtensile bond strength and nanoleakage. Dent Mater 23:862-870.

214. Reis A, Grandi V, Carlotto L, Bortoli G, Patzlaff R, Rodrigues Accorinte Mde L, Dourado Loguercio A (2005). Effect of smear layer thickness and acidity of self-etching solutions on early and long-term bond strength to dentin. J Dent 33:549-559.

215. Reis A, Klein-Junior CA, Accorinte Mde L, Grande RH, dos Santos CB, Loguercio AD (2009). Effects of adhesive temperature on the early and 6-month dentin bonding. J Dent 37:791-798.

216. Reis A, Loguercio AD, Carvalho RM, Grande RH (2004). Durability of resin dentin interfaces: effects of surface moisture and adhesive solvent component. Dent Mater 20:669-676.

217. Reis A, Pellizzaro A, Dal-Bianco K, Gones OM, Patzlaff R, Loguercio AD (2007). Impact of adhesive application to wet and dry dentin on long-term resin-dentin bond strengths. Oper Dent 32:380-387. 
218. Reis AF, Giannini M, Pereira PN (2008). Effects of a peripheral enamel bond on the long-term effectiveness of dentin bonding agents exposed to water in vitro. J Biomed Mater Res B Appl Biomater 85:10-17.

219. Ritter AV, Ghaname E, Leonard RH (2007). The influence of dental unit waterline cleaners on composite-to-dentin bond strengths. J Am Dent Assoc 138:985-91; quiz 1022-3.

220. Roberts HW, Vandewalle KS, Charlton DG, Leonard DL (2005). Effect of handpiece maintenance method on bond strength. Oper Dent 30:528-532.

221. Roberts S, Kim JR, Gu LS, Kim YK, Mitchell QM, Pashley DH, Tay FR (2009). The efficacy of different sealer removal protocols on bonding of self-etching adhesives to AH plus-contaminated dentin. J Endod 35:563-567.

222. Rocha PI, Borges AB, Rodrigues JR, Arrais CA, Giannini M (2006). Effect of dentinal surface preparation on bond strength of self-etching adhesive systems. Braz Oral Res 20:52-58.

223. Roh OD, Chung JH (2005). Micro-shear bond strength of five resin-based composites to dentin with five different dentin adhesives. Am J Dent 18:333-337.

224. Rolla JN, Mota EG, Oshima HM, Junior LH, Spohr AM (2006). Nd:YAG laser influence on microtensile bond strength of different adhesive systems for human dentin. Photomed Laser Surg 24:730-734.

225. Rosin C, Arana-Chavez VE, Netto NG, Luz MA (2005). Effects of cleaning agents on bond strength to dentin. Braz Oral Res 19:127-133.

226. Saboia VP, Nato F, Mazzoni A, Orsini G, Putignano A, Giannini M, Breschi L (2008). Adhesion of a two-step etch-and-rinse adhesive on collagen-depleted dentin. J Adhes Dent 10:419-422.

227. Sadek FT, Calheiros FC, Cardoso PE, Kawano Y, Tay F, Ferrari M (2008). Early and 24-hour bond strength and degree of conversion of etch-and-rinse and self-etch adhesives. Am J Dent 21:30-34.

228. Sadek FT, Goracci C, Cardoso PE, Tay FR, Ferrari M (2005). Microtensile bond strength of current dentin adhesives measured immediately and 24 hours after application. J Adhes Dent 7:297-302.

229. Sadr A, Ghasemi A, Shimada Y, Tagami J (2007). Effects of storage time and temperature on the properties of two self-etching systems. J Dent 35:218-225.

230. Sadr A, Shimada Y, Tagami J (2007). Effects of solvent drying time on micro-shear bond strength and mechanical properties of two self-etching adhesive systems. Dent Mater 23:1114-1119.

231. Salz U, Zimmermann J, Zeuner F, Moszner N (2005). Hydrolytic stability of self-etching adhesive systems. J Adhes Dent 7:107-116.

232. Sanabe ME, Kantovitz KR, Costa CA, Hebling J (2009). Effect of acid etching time on the degradation of resin-dentin bonds in primary teeth. Am J Dent 22:37-42.

233. Sardella TN, de Castro FL, Sanabe ME, Hebling J (2005). Shortening of primary dentin etching time and its implication on bond strength. J Dent 33:355-362.

234. Sattabanasuk V, Shimada Y, Tagami J (2006). Effects of saliva contamination on dentin bond strength using all-in-one adhesives. J Adhes Dent 8:311-318.

235. Sattabanasuk V, Shimada Y, Tagami J (2005). Bonding of resin to artificially carious dentin. J Adhes Dent 7:183-192.

236. Sattabanasuk V, Vachiramon V, Qian F, Armstrong SR (2007). Resin-dentin bond strength as related to different surface preparation methods. J Dent 35:467-475.

237. Sauro S, Mannocci F, Toledano M, Osorio R, Pashley DH, Watson TF (2009). EDTA or $\mathrm{H} 3 \mathrm{PO} 4 / \mathrm{NaOCl}$ dentine treatments may increase hybrid layers' resistance to degradation: a microtensile bond strength and confocal-micropermeability study. J Dent 37:279-288.

238. Sauro S, Pashley DH, Montanari M, Chersoni S, Carvalho RM, Toledano M, Osorio R, Tay FR, Prati $C$ (2007). Effect of simulated pulpal pressure on dentin permeability and adhesion of self-etch adhesives. Dent Mater 23:705-713.

239. Schmidlin PR, Siebenmann J, Kocher P, Seemann R, Attin T, Bindl A (2008). Effects of de- and remineralization of dentin on bond strengths yielded by one-, three-, and four-step adhesives. $J$ Adhes Dent 10:119-126.

240. Semeraro S, Mezzanzanica D, Spreafico D, Gagliani M, Re D, Tanaka T, Sidhu SK, Sano H (2006). Effect of different bur grinding on the bond strength of self-etching adhesives. Oper Dent 31:317323.

241. Sen D, Akgungor G (2005). Shear bond strengths of two composite core materials after using all-inone and single-bottle dentin adhesives. J Prosthodont 14:97-103.

242. Senawongse P, Harnirattisai C, Shimada Y, Tagami J (2004). Effective bond strength of current adhesive systems on deciduous and permanent dentin. Oper Dent 29:196-202.

243. Sengun A, Unlu N, Ozturk B, Ozer F (2005). Microtensile bond strength of two resin composite materials placed with direct and indirect techniques under simulated pulpal pressure. J Adhes Dent 7:197-202.

244. Sensi LG, Lopes GC, Monteiro S Jr, Baratieri LN, Vieira LC (2005). Dentin bond strength of selfetching primers/adhesives. Oper Dent 30:63-68.

245. Shafiei F, Memarpour M (2009). Effect of repeated use on dentin bond strength of two adhesive systems: all-in-one and one-bottle. Indian J Dent Res 20:180-184.

246. Shinkai K, Suzuki S, Katoh Y (2006). Effect of air-blowing variables on bond strength of all-in-one adhesives to bovine dentin. Dent Mater J 25:664-668. 
247. Shinohara MS, Peris AR, Rodrigues JA, Pimenta LA, Ambrosano GM (2004). The effect of nonvital bleaching on the shear bond strength of composite resin using three adhesive systems. $J$ Adhes Dent 6:205-209.

248. Shirai K, De Munck J, Yoshida Y, Inoue S, Lambrechts P, Suzuki K, Shintani H, Van Meerbeek B (2005). Effect of cavity configuration and aging on the bonding effectiveness of six adhesives to dentin. Dent Mater 21:110-124.

249. Sidhu SK, Omata Y, Tanaka T, Koshiro K, Spreafico D, Semeraro S, Mezzanzanica D, Sano H (2007). Bonding characteristics of newly developed all-in-one adhesives. J Biomed Mater Res $B$ Appl Biomater 80:297-303.

250. Silva EM, Duarte PB, Poskus LT, Barcellos AA, Guimaraes JG (2007). Nanoleakage and microshear bond strength in deproteinized human dentin. J Biomed Mater Res B Appl Biomater 81:336-342.

251. Silva NR, Calamia CS, Coelho PG, Carrilho MR, Carvalho RM, Caufield P, Thompson VP (2006). Effect of 2\% iodine disinfecting solution on bond strength to dentin. J Appl Oral Sci 14:399-404.

252. Silva NR, Calamia CS, Harsono M, Carvalho RM, Pegoraro LF, Fernandes CA, Vieira AC, Thompson VP (2006). Bond angle effects on microtensile bonds: laboratory and FEA comparison. Dent Mater 22:314-324.

253. Sonoda H, Banerjee A, Sherriff M, Tagami J, Watson TF (2005). An in vitro investigation of microtensile bond strengths of two dentine adhesives to caries-affected dentine. J Dent 33:335-342.

254. Souza-Zaroni WC, Seixas LC, Ciccone-Nogueira JC, Chimello DT, Palma-Dibb RG (2007). Tensile bond strength of different adhesive systems to enamel and dentin. Braz Dent J 18:124-128.

255. Spreafico D, Semeraro S, Mezzanzanica D, Re D, Gagliani M, Tanaka T, Sano H, Sidhu SK (2006) The effect of the air-blowing step on the technique sensitivity of four different adhesive systems. $J$ Dent 34:237-244.

256. Stanislawczuk R, Amaral RC, Zander-Grande C, Gagler D, Reis A, Loguercio AD (2009). Chlorhexidine-containing acid conditioner preserves the longevity of resin-dentin bonds. Oper Dent 34:481-490

257. Thomsen KB, Peutzfeldt A (2007). Resin composites: strength of the bond to dentin versus mechanical properties. Clin Oral Investig 11:45-49.

258. Toledano M, Osorio R, Albaladejo A, Aguilera FS, Osorio E (2006). Differential effect of in vitro degradation on resin-dentin bonds produced by self-etch versus total-etch adhesives. $J$ Biomed Mater Res A 77:128-135.

259. Toledano M, Osorio R, Albaladejo A, Aguilera FS, Tay FR, Ferrari M (2006). Effect of cyclic loading on the microtensile bond strengths of total-etch and self-etch adhesives. Oper Dent 31:25-32.

260. Toledano M, Osorio R, Moreira MA, Cabrerizo-Vilchez MA, Gea P, Tay FR, Pashley DH (2004). Effect of the hydration status of the smear layer on the wettability and bond strength of a self-etching primer to dentin. Am J Dent 17:310-314.

261. Toledano M, Osorio R, Osorio E, Aguilera FS, Yamauti M, Pashley DH, Tay F (2007). Effect of bacterial collagenase on resin-dentin bonds degradation. J Mater Sci Mater Med 18:2355-2361.

262. Toledano M, Osorio R, Osorio E, Aguilera FS, Yamauti M, Pashley DH, Tay F (2007). Durability of resin-dentin bonds: effects of direct/indirect exposure and storage media. Dent Mater 23:885-892.

263. Torkabadi S, Nakajima M, Ikeda M, Foxton RM, Tagami J (2008). Bonding durability of HEMA-free and HEMA-containing one-step adhesives to dentine surrounded by bonded enamel. J Dent 36:8086.

264. Torres CP, Ciccone JC, Ramos RP, Corona SA, Palma-Dibb RG, Borsatto MC (2005). Tensile bond strength of self-etching adhesive systems to primary dentin. Am J Dent 18:327-332.

265. Torres CP, Corona SA, Ramos RP, Palma-Dibb RG, Borsatto MC (2004). Bond strength of selfetching primer and total-etch adhesive systems to primary dentin. J Dent Child (Chic) 71:131-134.

266. Torres CP, Gomes-Silva JM, Borsatto MC, Barroso JM, Pecora JD, Palma-Dibb RG (2009). Shear bond strength of self-etching and total-etch adhesive systems to Er:YAG laser-irradiated primary dentin. J Dent Child (Chic) 76:67-73.

267. Tosun G, Koyuturk AE, Sener Y, Sengun A (2008). Bond strength of two total-etching bonding systems on caries-affected and sound primary teeth dentin. Int J Paediatr Dent 18:62-69.

268. Uekusa S, Tsubota K, Tonegawa M, Tsuchiya H, Iwasa M, Kawamoto R, Takamizawa T, Miyazaki M (2007). Microtensile bond strengths of single-step self-etch adhesive systems to bovine dentin. $J$ Oral Sci 49:183-189.

269. Uekusa S, Yamaguchi K, Miyazaki M, Tsubota K, Kurokawa H, Hosoya Y (2006). Bonding efficacy of single-step self-etch systems to sound primary and permanent tooth dentin. Oper Dent 31:569576.

270. Vachiramon V, Vargas MA, Pashley DH, Tay FR, Geraldeli S, Qian F, Armstrong SR (2008). Effects of oxalate on dentin bond after 3-month simulated pulpal pressure. J Dent 36:178-185.

271. Van Landuyt KL, De Munck J, Snauwaert J, Coutinho E, Poitevin A, Yoshida Y, Inoue S, Peumans M, Suzuki K, Lambrechts P, Van Meerbeek B (2005). Monomer-solvent phase separation in onestep self-etch adhesives. J Dent Res 84:183-188.

272. Van Landuyt KL, Mine A, De Munck J, Jaecques S, Peumans M, Lambrechts P, Van Meerbeek B (2009). Are one-step adhesives easier to use and better performing? Multifactorial assessment of contemporary one-step self-etching adhesives. J Adhes Dent 11:175-190. 
273. Van Landuyt KL, Peumans M, De Munck J, Lambrechts P, Van Meerbeek B (2006). Extension of a one-step self-etch adhesive into a multi-step adhesive. Dent Mater 22:533-544.

274. Velasquez LM, Sergent RS, Burgess JO, Mercante DE (2006). Effect of placement agitation and placement time on the shear bond strength of 3 self-etching adhesives. Oper Dent 31:426-430.

275. Visintini E, Mazzoni A, Vita F, Pasquantonio G, Cadenaro M, Di Lenarda R, Breschi L (2008). Effects of thermocycling and use of ElectroBond on microtensile strength and nanoleakage using commercial one-step self-etch adhesives. Eur J Oral Sci 116:564-570.

276. Waidyasekera PG, Nikaido T, Weerasinghe DD, Tagami J (2007). Bonding of acid-etch and selfetch adhesives to human fluorosed dentine. J Dent 35:915-922.

277. Walter R, Miguez PA, Swift EJ Jr, Pereira PN (2008). Long-term bond strengths to dentin treated with different re-wetting solutions. Am J Dent 21:143-147.

278. Wattanawongpitak N, Nakajima M, Ikeda M, Foxton RM, Tagami J (2009). Microtensile bond strength of etch-and-rinse and self-etching adhesives to intrapulpal dentin after endodontic irrigation and setting of root canal sealer. J Adhes Dent 11:57-64.

279. Wei S, Sadr A, Shimada Y, Tagami J (2008). Effect of caries-affected dentin hardness on the shear bond strength of current adhesives. J Adhes Dent 10:431-440.

280. Yamada M, Miyazaki M, Onose H, Moore BK (2005). Influence of interchanging self-etching primers and bonding agents on bond strengths to bovine dentin. Am J Dent 18:155-159.

281. Yamaguchi K, Miyazaki M, Takamizawa T, Tsubota K, Rikuta A (2006). Influence of crosshead speed on micro-tensile bond strength of two-step adhesive systems. Dent Mater 22:420-425.

282. Yamamoto A, Tsubota K, Takamizawa T, Kurokawa H, Rikuta A, Ando S, Takigawa T, Kuroda T, Miyazaki M (2006). Influence of light intensity on dentin bond strength of self-etch systems. J Oral Sci 48:21-26.

283. Yamazaki PC, Bedran-Russo AK, Pereira PN (2008). Importance of the hybrid layer on the bond strength of restorations subjected to cyclic loading. J Biomed Mater Res B Appl Biomater 84:291297.

284. Yaseen SM, Subba Reddy VV (2009). Comparative evaluation of shear bond strength of two selfetching adhesives (sixth and seventh generation) on dentin of primary and permanent teeth: an in vitro study. J Indian Soc Pedod Prev Dent 27:33-38.

285. Yazici AR, Celik C, Ozgunaltay G, Dayangac B (2007). Bond strength of different adhesive systems to dental hard tissues. Oper Dent 32:166-172.

286. Yazici AR, Karaman E, Ertan A, Ozgunaltay G, Dayangac B (2009). Effect of different pretreatment methods on dentin bond strength of a one-step self-etch adhesive. J Contemp Dent Pract 10:41-48.

287. Yazici E, Gurgan S, Gutknecht N, Imazato S (2010). Effects of erbium:yttrium-aluminum-garnet and neodymium:yttrium-aluminum-garnet laser hypersensitivity treatment parameters on the bond strength of self-etch adhesives. Lasers Med Sci 25:511-516.

288. Yesilyurt C, Bulucu B (2006). Bond strength of total-etch and self-etch dentin adhesive systems on peripheral and central dentinal tissue: a microtensile bond strength test. J Contemp Dent Pract 7:2636.

289. Yildirim S, Tosun G, Koyuturk AE, Sener Y, Sengun A, Ozer F, Imazato S (2008). Microtensile and microshear bond strength of an antibacterial self-etching system to primary tooth dentin. Eur J Dent 2:11-17

290. Yiu CK, Hiraishi N, King NM, Tay FR (2008). Effect of dentinal surface preparation on bond strength of self-etching adhesives. J Adhes Dent 10:173-182.

291. Yiu CK, King NM, Suh BI, Sharp LJ, Carvalho RM, Pashley DH, Tay FR (2005). Incompatibility of oxalate desensitizers with acidic, fluoride-containing total-etch adhesives. J Dent Res 84:730-735.

292. Yokota H, Kubo S, Yokota H, Ohsawa M, Hayashi Y (2006). Effect of a caries-detecting solution on the tensile bond strength of four dentin adhesive systems. Dent Mater J 25:66-74.

293. Yoo HM, Oh TS, Pereira PN (2006). Effect of saliva contamination on the microshear bond strength of one-step self-etching adhesive systems to dentin. Oper Dent 31:127-134.

294. Yoo HM, Pereira PN (2006). Effect of blood contamination with 1-step self-etching adhesives on microtensile bond strength to dentin. Oper Dent 31:660-665.

295. Yu L, Liu JM, Wang XY, Gao XJ (2009). [Evaluation of shear bond strengths of self-etching and total-etching dental adhesives to enamel and dentin]. Zhonghua Kou Qiang Yi Xue Za Zhi 44:165167.

296. Yurdaguven H, Tanalp J, Toydemir B, Mohseni K, Soyman M, Bayirli G (2009). The effect of endodontic irrigants on the microtensile bond strength of dentin adhesives. J Endod 35:1259-1263.

297. Zhang ZX, Huang C, Zheng TL, Wang S, Cheng XR (2005). Effects of residual water on microtensile bond strength of one-bottle dentin adhesive systems with different solvent bases. Chin Med J (Engl) 118:1623-1628.

298. Zorba YO, Erdemir A, Ercan E, Eldeniz AU, Kalaycioglu B, Ulker M (2010). The effects of three different desensitizing agents on the shear bond strength of composite resin bonding agents. $J$ Mech Behav Biomed Mater 3:399-404. 
Research Coordination Office

Huis Bethlehem

Schapenstraat 34

B-3000 Leuven

Tel.: +32 16324065

Fax: +3216324198

onderzoek@kuleuven.be

www.kuleuven.be 